\title{
A Stereospecific Synthesis of Chiral Cyclic Sulfinamides
}

\author{
Michael Harmata* and Pinguan Zheng \\ Department of Chemistry, University of Missouri-Columbia \\ Columbia, Missouri 65211
}

TABLE OF CONTENT

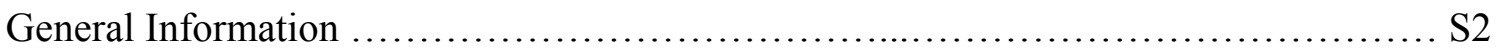

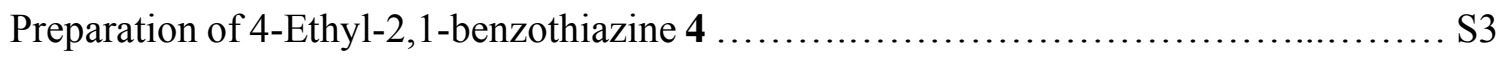

Typical Procedure of Dephenylation Reaction ................................... S4

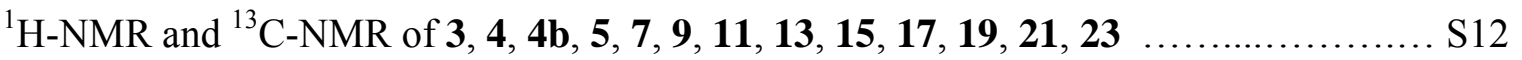




\section{General Information}

All reactions were carried out under an atmosphere of nitrogen or argon in flamed-dried glassware. Ethyl ether and THF were distilled over sodium-benzophenone. Acetonitrile was distilled over cacium hydride.

All commercial grade reagents and solvents were used, unless otherwise noted. Lithium triethylborohydride (1.0M in THF) and resublimed iodine were purchased from Acros chemical company. Enantiomerically pure 4a was prepared from commercially available 2-bromo benzaldehyde employing our benzothiazine synthesis. ${ }^{1} \mathbf{6}^{2}, \mathbf{8}^{2,3}, \mathbf{1 0}^{2,3}$ and $\mathbf{1 8}^{4}$ were synthesized as literatures reported. The synthesis of $2,12,14,16,20$ and 22 will be reported soon.

Chromatographic separations were carried out using Silicycle ultra pure silica gel (230400 mesh). Analytical thin chromatography was performed on EM reagent $0.25 \mathrm{~nm}$ silica gel 60-F plates with F-254 indicator. Visulization was accomplished with UV light. Melting points were measured with a Fisher-Johns melting point apparatus. Infrared spectra were recorded on a Thermo Nicolet NEXUS 670 FT-IR spectrometer. Optical rotations were measured on a Jasco DIP-370 digital polarimeter with a sodium lamp and are reported as follows: $[\alpha]_{\mathrm{D}}^{25}(\mathrm{c} / 100 \mathrm{~mL}$, solvent). HRMS was carried out using Bruker 12 Tesla FRICR-MS with an Apollo II ion source in Old Dominion University.

Quantitative GC-MS analysis was performed on an Agilent 5973N Massive Selective Detector interfaced to an Agilent 6890 GC System equipped with an HP capillary column (HP-624, 27.8m X $0.25 \mathrm{~mm}$ ). $n$-Decane was utilized as internal standard. GC conditions were as followed: flow rate: $1 \mathrm{ml} / \mathrm{min} ; 70{ }^{\circ} \mathrm{C}, 5 \mathrm{~min}, 10{ }^{\circ} \mathrm{C} / \mathrm{min}, 200{ }^{\circ} \mathrm{C}, 1 \mathrm{~min}$.

${ }^{1} \mathrm{H}-\mathrm{NMR}$ were recorded on a Bruker ARX-250 (250 MHz), DRX-300 (300 MHz), DRX$500(500 \mathrm{MHz})$ spectrometer and are reported in ppm $(\delta)$ from tetramethylsilane (TMS: $\delta$ $0.0 \mathrm{ppm}$ ). Data are reported as follows: chemical shift, multiplicity ( $\mathrm{s}=$ singlet, $\mathrm{d}=$ doublet, $\mathrm{t}=$ triplet, $\mathrm{q}=$ quartet, $\mathrm{m}=$ multiplet, $\mathrm{dd}=$ doublet of doublet, $\mathrm{ddd}=$ doublet of doublet of doublet), coupling constants $(\mathrm{Hz})$ and integration. ${ }^{13} \mathrm{C}-\mathrm{NMR}$ spectra were 
recorded on a Bruker ARX-250 (62.5 MHz), DRX-300 (75 MHz), DRX-500 (125 MHz) spectrometer with complete proton decoupling. Chemical shifts are reported in ppm from tetramethylsilane with solvent resonance as the internal standard $\left(\mathrm{CDCl}_{3}: \delta 77.0 \mathrm{ppm}\right)$.

\section{Preparation of 4-ethyl-2,1-benzothiazine 4}<smiles>CC(=O)CC1C[S+]([O-])(c2ccccc2)=Nc2ccccc21</smiles>

4a

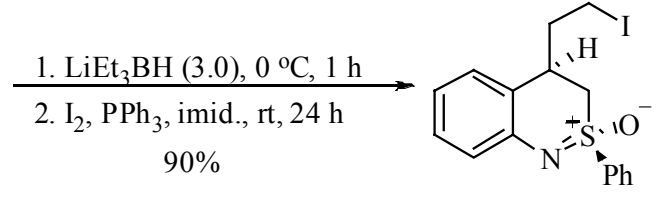

4b

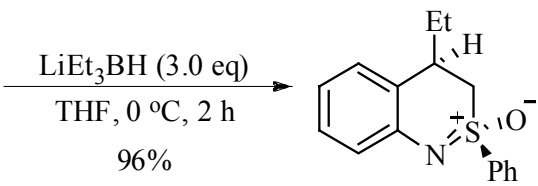

4a $(1.2 \mathrm{~g}, 4.0 \mathrm{mmol})$ was dissolved in $70 \mathrm{~mL}$ THF. The solution was cooled to $0{ }^{\circ} \mathrm{C}$ before it was dropwisely added lithium triethylborohydride (1.0 M in THF solution, 12 $\mathrm{ml}, 12 \mathrm{mmol})$. After the addition, the stirring was continued for another 1 hour. The reaction was quenched by sequential addition of methanol, $1 \mathrm{~N} \mathrm{NaOH}$ solution and hydrogen peroxide solution. Extract the reaction mixture with $\mathrm{CH}_{2} \mathrm{Cl}_{2}$ (3 times) and dry over $\mathrm{MgSO}_{4}$. After the removal of solvent by rotary evaporization, crude product was obtained in $1.17 \mathrm{~g}$, which was used for next step without further purification.

To a cold solution of crude alcohol (662 mg), triphenylphospine (1.2 g, $4.6 \mathrm{mmol})$ and imidazole $(312 \mathrm{mg}, 4.6 \mathrm{mmol})$ in acetonitrile $(5.0 \mathrm{~mL})$ and ether $(20 \mathrm{~mL})$, was slowly added iodine (1.16 g, $4.6 \mathrm{mmol}$ ) resulting in a pale yellow suspension. After being stirred for overnight, the reaction mixture was diluted with ether and sequentially washed with saturated aqueous $\mathrm{Na}_{2} \mathrm{~S}_{2} \mathrm{O}_{3}$, saturated $\mathrm{CuSO}_{4}$ and water. The organic layer was dried briefly over $\mathrm{MgSO}_{4}$, filtered and concentrated to give crude product. Silica gel column chromatography (30\% EtOAc/Hexanes) gave pure iodide $\mathbf{4 b} 820 \mathrm{mg}$ (90\% yield) as pale yellow solid, mp: $103-4{ }^{\circ} \mathrm{C}$; IR: $v$ 2929.4, 1482.8, 1442.0, 1017.0, $747.3 \mathrm{~cm}^{-1}$; ${ }^{1} \mathrm{H}-\mathrm{NMR}$ $\left(500 \mathrm{MHz}, \mathrm{CDCl}_{3}\right): \delta 8.08(\mathrm{~d}, J=7.5 \mathrm{~Hz}, 2 \mathrm{H}), 7.68(\mathrm{t}, J=7.2 \mathrm{~Hz}, 1 \mathrm{H}), 7.59(\mathrm{t}, J=7.5$ Hz, 2H), 7.23-7.27 (m, 1H), 7.17 (d, $J=7.0 \mathrm{~Hz}, 1 \mathrm{H}), 7.14$ (d, $J=7.5 \mathrm{~Hz}, 1 \mathrm{H}), 6.95$ (t, $J$ $=7.2 \mathrm{~Hz}, 1 \mathrm{H}), 3.52(\mathrm{dd}, J=8.5,13.0 \mathrm{~Hz}, 1 \mathrm{H}), 3.47-3.49(\mathrm{~m}, 1 \mathrm{H}), 3.23(\mathrm{dd}, J=5.0,13.5$ $\mathrm{Hz}, 1 \mathrm{H}), 3.03-3.14(\mathrm{~m}, 2 \mathrm{H}), 2.47-2.54(\mathrm{~m}, 1 \mathrm{H}), 2.08-2.14(\mathrm{~m}, 1 \mathrm{H}) ;{ }^{13} \mathrm{C}-\mathrm{NMR}(75 \mathrm{MHz}$, $\left.\mathrm{CDCl}_{3}\right): \delta 144.7,139.3,133.8,129.5,128.7,126.2,124.5,124.1,121.1,52.5,36.0,35.7$, $1.96 ;[\alpha]^{25}=-7.83\left(c 2.92, \mathrm{CHCl}_{3}\right)$. 
To the THF $(40 \mathrm{~mL})$ solution of iodide $4 \mathbf{b}(820 \mathrm{mg})$ at $0{ }^{\circ} \mathrm{C}$, was dropwise added lithium triethylborohydride (1.0M in THF solution, $6.2 \mathrm{~mL}, 6.2 \mathrm{mmol})$. The stirring was continued for another $2.0 \mathrm{~h}$ before it was quenched by the sequential addition of methanol, $1 \mathrm{~N} \mathrm{NaOH}$ and hydrogen peroxide solution. The reaction mixture was extracted with $\mathrm{CH}_{2} \mathrm{Cl}_{2}$ (3 times) and dry over $\mathrm{MgSO}_{4}$. After the removal of solvent by rotary evaporation, crude product was subjected to silica gel column chromatography $(30 \%$ EtOAc in hexanes). Compound 4 was isolated in $540 \mathrm{mg}$ ( $96 \%$ yield).

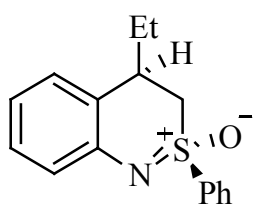

4

Yellow solid, mp: 102-3 ${ }^{\circ} \mathrm{C}$; IR: $v$ 2966.1, 1597.2, 1474.6, 1442.0, 1270.3, 1204.9, 1111.0, 1012.9, $747.3 \mathrm{~cm}^{-1}$; ${ }^{1} \mathrm{H}-\mathrm{NMR}\left(250 \mathrm{MHz}, \mathrm{CDCl}_{3}\right): \delta 8.06-8.11(\mathrm{~m}, 2 \mathrm{H}), 7.54-7.70$ (m, 3H), $7.23(\mathrm{t}, J=7.2 \mathrm{~Hz}, 2 \mathrm{H}), 7.12-7.16(\mathrm{~m}, 1 \mathrm{H}), 6.94(\mathrm{td}, J=7.4,1.5 \mathrm{~Hz}, 1 \mathrm{H}), 3.37-$ $3.51(\mathrm{~m}, 2 \mathrm{H}), 2.92(\mathrm{t}, J=11.5 \mathrm{~Hz}, 1 \mathrm{H}), 2.09-2.21(\mathrm{~m}, 1 \mathrm{H}), 1.84(\mathrm{td}, J=14.2,7.1 \mathrm{~Hz}, 1 \mathrm{H})$, $0.98(\mathrm{t}, J=7.4 \mathrm{~Hz}, 3 \mathrm{H}) ;{ }^{13} \mathrm{C}-\mathrm{NMR}\left(62.5 \mathrm{MHz}, \mathrm{CDCl}_{3}\right): \delta 145.2,139.1,133.7,129.3$, $128.8,128.1,125.6,125.4,123.7,120.5,51.3,34.6,24.2,10.5$; HRMS cacled for $\mathrm{C}_{16} \mathrm{H}_{17} \mathrm{NOSNa}^{+}[\mathrm{M}+\mathrm{Na}]^{+} 294.0923$, found 294.0919; $[\alpha]^{25}=+3.13\left(\right.$ c $\left.0.83, \mathrm{CHCl}_{3}\right)$.

\section{Typical Procedure of Dephenylation Reaction}

To the THF $(6.0 \mathrm{~mL})$ solution of $4(0.3 \mathrm{mmol})$ at $0{ }^{\circ} \mathrm{C}$, was dropwisely added lithium triethylborohydride $(1.0 \mathrm{M}$ in THF solution, $0.9 \mathrm{~mL}, 0.9 \mathrm{mmol})$. The resulting mixture was refluxed for certain hours. The reaction was monitored by TLC analysis. When the reaction was complete, the mixture was cooled to room temperature and quenched by the sequential addition of methanol, $1 \mathrm{~N} \mathrm{NaOH}$ and hydrogen peroxide solution. The reaction mixture was extracted with $\mathrm{CH}_{2} \mathrm{Cl}_{2}$ (3 times) and dried over $\mathrm{MgSO}_{4}$. After the removal of solvent by rotary evaporation, crude product was subjected to silica gel column chromatography. 


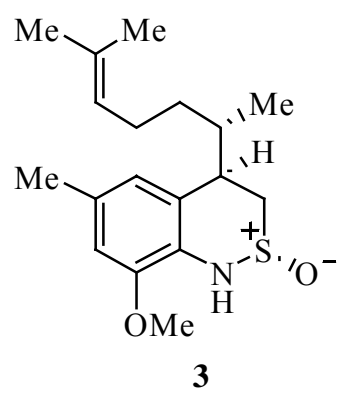

3: yellow liquid, $57 \%$ yield (65\% yield based on recovered starting material), silica gel column chromatography (30\% EtOAc in Hexanes); IR: v 3252.2, 2917.1, 1585..0, 1482.8, 1458.3, 1082.4, 878.0, $829.0 \mathrm{~cm}^{-1} ;{ }^{1} \mathrm{H}-\mathrm{NMR}\left(300 \mathrm{MHz}, \mathrm{CDCl}_{3}\right): \delta 6.86(\mathrm{~s}, 1 \mathrm{H}), 6.74(\mathrm{~s}$, 1H), 6.56, (s, 1H), $5.16(\mathrm{td}, J=7.2,1.2 \mathrm{~Hz}, 1 \mathrm{H}), 3.83$ (s, $3 \mathrm{H}), 3.63$ (dt, $J=13.0,4.0 \mathrm{~Hz}$, $1 \mathrm{H}), 2.90(\mathrm{ddd}, J=12.8,4.7,2.4 \mathrm{~Hz}, 1 \mathrm{H}), 2.60(\mathrm{t}, J=13.0 \mathrm{~Hz}, 1 \mathrm{H}), 2.56-2.60(\mathrm{~m}, 1 \mathrm{H})$, $2.31(\mathrm{~s}, 3 \mathrm{H}), 2.05-2.13(\mathrm{~m}, 2 \mathrm{H}), 1.72(\mathrm{~s}, 3 \mathrm{H}), 1.64(\mathrm{~s}, 3 \mathrm{H}), 1.36-1.48(\mathrm{~m}, 2 \mathrm{H}), 0.82(\mathrm{~d}, J=$ $6.8 \mathrm{~Hz}, 3 \mathrm{H}) ;{ }^{13} \mathrm{C}-\mathrm{NMR}\left(75 \mathrm{MHz}, \mathrm{CDCl}_{3}\right): \delta 147.4,131.9,130.8,125.4,123.9,122.6$, 118.8, 109.1, 55.6, 44.2, 35.0, 32.5, 29.7, 26.0, 25.7, 21.5, 17.7, 14.6; HRMS caclcd for $\mathrm{C}_{18} \mathrm{H}_{27} \mathrm{NO}_{2} \mathrm{SNa}^{+}[\mathrm{M}+\mathrm{Na}]^{+}$344.1654, found 344.1647.

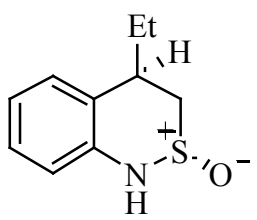

5

5: Colorless crystal (recrystallized from Hexane-EtOAc), 71\% yield, silica gel column chromatography (50\% EtOAc/Hexanes); mp: 112-3 ${ }^{\circ} \mathrm{C}$; IR: $v$ 3170.4, 1470.6, 1037.4, 898.5, $747.3 \mathrm{~cm}^{-1}$; ${ }^{1} \mathrm{H}-\mathrm{NMR}\left(500 \mathrm{MHz}, \mathrm{CDCl}_{3}\right): \delta 7.31(\mathrm{~d}, J=7.5 \mathrm{~Hz}, 1 \mathrm{H}), 7.05(\mathrm{t}, J=$ $7.5 \mathrm{~Hz}, 1 \mathrm{H}), 6.95(\mathrm{td}, J=7.5,1.0 \mathrm{~Hz}, 1 \mathrm{H}), 6.80(\mathrm{~s}, 1 \mathrm{H}), 6.58(\mathrm{td}, J=8.0,1.0 \mathrm{~Hz}, 1 \mathrm{H})$, 3.59-3.65 (m, 1H), $3.01(\mathrm{ddd}, J=13.0,4.0,2.0 \mathrm{~Hz}, 1 \mathrm{H}), 2.64(\mathrm{t}, J=13.0 \mathrm{~Hz}, 1 \mathrm{H}), 2.11$ $2.17(\mathrm{~m}, 1 \mathrm{H}), 1.99(\mathrm{td}, J=15.0,7.5 \mathrm{~Hz}, 1 \mathrm{H}), 1.00(\mathrm{t}, J=7.5 \mathrm{~Hz}, 3 \mathrm{H}) ;{ }^{13} \mathrm{C}-\mathrm{NMR}(125$ $\mathrm{MHz}_{\mathrm{CDCl}}$ ): $\delta 135.4,127.4,126.6,125.3,121.9,117.4,48.5,26.4,24.2,10.0$; HRMS cacled for $\mathrm{C}_{10} \mathrm{H}_{13} \mathrm{NOSNa}^{+}[\mathrm{M}+\mathrm{Na}]^{+} 218.0610$, found 218.0606; $[\alpha]^{25}{ }_{\mathrm{D}}=-61.2(c 0.84$, $\left.\mathrm{CHCl}_{3}\right)$. 


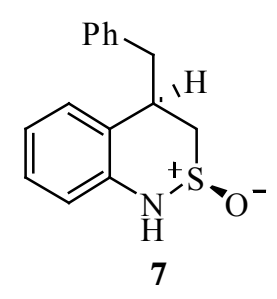

7: Off-white solid, 99\% yield, silica gel column chromatography (50\% EtOAc/Hexanes); mp: 153-4 ${ }^{\circ} \mathrm{C}$; IR: v 3190.9, 1462.4, 1049.7, $743.2 \mathrm{~cm}^{-1} ;{ }^{1} \mathrm{H}-\mathrm{NMR}\left(250 \mathrm{MHz}, \mathrm{CDCl}_{3}\right): \delta$ 7.46 (s, 1H), 7.26-7.38 (m, 5H), 7.22 (d, $J=7.5 \mathrm{~Hz}, 1 \mathrm{H}), 7.14$ (td, $J=7.5,1.4 \mathrm{~Hz}, 1 \mathrm{H})$, $7.00(\mathrm{td}, J=7.4,1.2 \mathrm{~Hz}, 1 \mathrm{H}), 6.78(\mathrm{dd}, J=7.8,1.1 \mathrm{~Hz}, 1 \mathrm{H}), 3.63(\mathrm{dd}, J=12.6,10.7 \mathrm{~Hz}$, 1H), 3.30-3.40 (m, 1H), 3.24 (dd, $J=12.6,5.2 \mathrm{~Hz}, 1 \mathrm{H}), 3.04$ (ddd, $J=13.7 .3 .4,1.8 \mathrm{~Hz}$, $1 \mathrm{H}), 2.77(\mathrm{dd}, J=13.7,6.0 \mathrm{~Hz}, 1 \mathrm{H}) ;{ }^{13} \mathrm{C}-\mathrm{NMR}\left(62.5 \mathrm{MHz}, \mathrm{CDCl}_{3}\right): \delta 139.5,134.6,129.6$, 129.3, 128.5, 127.9, 126.3, 125.7, 122.1, 118.2, 46.5, 41.5, 35.9; HRMS caclcd for $\mathrm{C}_{15} \mathrm{H}_{15} \mathrm{NOSNa}^{+}[\mathrm{M}+\mathrm{Na}]^{+} 280.0766$, found 280.0760 .

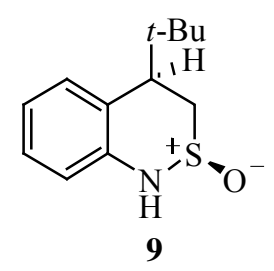

9: White solid, 96\% yield, silica gel column chromatography (50\% EtOAc/Hexanes); mp: 179-80 ${ }^{\circ} \mathrm{C}$; IR: $v$ 3182.7, 2958.0, 1474.6, 1061.9, 890.3, $751.4 \mathrm{~cm}^{-1} ;{ }^{1} \mathrm{H}-\mathrm{NMR}(500 \mathrm{MHz}$, $\left.\mathrm{CDCl}_{3}\right): \delta 7.30(\mathrm{~d}, J=7.5 \mathrm{~Hz}, 1 \mathrm{H}), 7.19(\mathrm{td}, J=7.5,1.0 \mathrm{~Hz}, 1 \mathrm{H}), 7.00(\mathrm{td}, J=7.5,1,0 \mathrm{~Hz}$, $1 \mathrm{H}), 6.79(\mathrm{~d}, J=8.0 \mathrm{~Hz}, 1 \mathrm{H}), 3.21(\mathrm{dd}, J=14.3,5.3 \mathrm{~Hz}, 1 \mathrm{H}), 3.06$ (dd, $J=14.2,6.4 \mathrm{~Hz}$, $1 \mathrm{H}), 2.90(\mathrm{t}, J=5.8 \mathrm{~Hz}, 1 \mathrm{H}), 1.19(\mathrm{~s}, 9 \mathrm{H}) ;{ }^{13} \mathrm{C}-\mathrm{NMR}\left(125 \mathrm{MHz}, \mathrm{CDCl}_{3}\right): \delta 137.5,129.5$, 127.6, 126.3, 121.9, 118.2, 51.8, 45.7, 34.1, 28.7; HRMS cacled for $\mathrm{C}_{12} \mathrm{H}_{17} \mathrm{NOSNa}^{+}[\mathrm{M}+$ $\mathrm{Na}]^{+}$246.0923, found 246.0916 .

11: $90 \%$ yield, a mixture of cis and trans isomers (d.r.: 1.7: 1.0), which were partially separated by silica gel column chromatography (50\% EtOAc/Hexanes). 


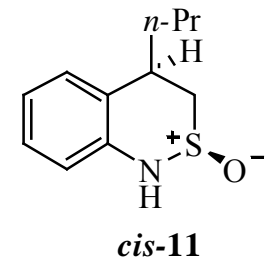

$1^{\text {st }}$ fraction, major isomer (cis): off-white solid, mp: $159-60{ }^{\circ} \mathrm{C}$; IR: $v$ 3166.4, 2958.0, 1462.4, 1041.5, 902.6, $739.1 \mathrm{~cm}^{-1} ;{ }^{1} \mathrm{H}-\mathrm{NMR}\left(250 \mathrm{MHz}, \mathrm{CDCl}_{3}\right): \delta 7.18$ (d, $J=6.6 \mathrm{~Hz}$, 1H), 7.12 (dd, $J=7.6,1.6 \mathrm{~Hz}, 1 \mathrm{H}), 7.00$ (td, $J=7.4,1,2 \mathrm{~Hz}, 1 \mathrm{H}), 6.92$ (s, 1H), 6.75 (dd, $J=7.8,1.1 \mathrm{~Hz}, 1 \mathrm{H}), 2.96-3.16(\mathrm{~m}, 3 \mathrm{H}), 2.11-2.18(\mathrm{~m}, 1 \mathrm{H}), 1.91-1.98(\mathrm{~m}, 1 \mathrm{H}), 1.42-1.62$ (m, 2H), 0.99 (t, $J=7.3 \mathrm{~Hz}, 3 \mathrm{H}) ;{ }^{13} \mathrm{C}-\mathrm{NMR}\left(62.5 \mathrm{MHz}, \mathrm{CDCl}_{3}\right): \delta 134.8,128.3,127.7$, 127.6, 122.3, 118.2, 50.3, 36.4, 33.8, 20.9, 13.8; HRMS caclcd for $\mathrm{C}_{11} \mathrm{H}_{15} \mathrm{NOSNa}^{+}[\mathrm{M}+$ $\mathrm{Na}]^{+} 232.0766$, found 232.0764 .

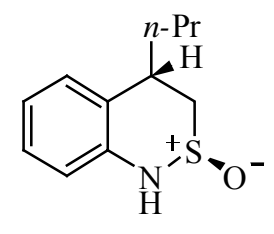

trans-11

$2^{\text {nd }}$ fraction, minor isomer (trans): white solid, mp: 105-6 ${ }^{\circ} \mathrm{C}$; IR: $v$ 3154.1, 2945.7, 1470.6, 1041.5, 910.7, $747.3 \mathrm{~cm}^{-1} ;{ }^{1} \mathrm{H}-\mathrm{NMR}\left(500 \mathrm{MHz}, \mathrm{CDCl}_{3}\right): \delta 7.31$ (d, $J=7.5 \mathrm{~Hz}$, 1H), 7.08 (t, $J=7.5 \mathrm{~Hz}, 1 \mathrm{H}), 7.02(\mathrm{~s}, 1 \mathrm{H}), 6.97$ (t, $J=7.5 \mathrm{~Hz}, 1 \mathrm{H}), 6.65$ (d, $J=8.0 \mathrm{~Hz}$, $1 \mathrm{H}), 3.60-3.66(\mathrm{~m}, 1 \mathrm{H}), 3.01(\mathrm{ddd}, J=13.0,4.0,2.0 \mathrm{~Hz}, 1 \mathrm{H}), 2.61(\mathrm{t}, J=13.0 \mathrm{~Hz}, 1 \mathrm{H})$, 2.03-2.11 (m, $1 \mathrm{H}), 1.83-1.91(\mathrm{~m}, 1 \mathrm{H}), 1.45-1.52(\mathrm{~m}, 1 \mathrm{H}), 1.32-1.39(\mathrm{~m}, 1 \mathrm{H}), 1.01(\mathrm{t}, J=$ $7.5 \mathrm{~Hz}, 3 \mathrm{H}) ;{ }^{13} \mathrm{C}-\mathrm{NMR}\left(125 \mathrm{MHz}, \mathrm{CDCl}_{3}\right): \delta 135.4,127.3,126.5,125.8,121.9,117.4$, 48.9, 33.8, 25.3, 19.1, 14.2; HRMS caclcd for $\mathrm{C}_{11} \mathrm{H}_{15} \mathrm{NOSNa}^{+}[\mathrm{M}+\mathrm{Na}]^{+} 232.0766$, found 232.0764 .

13: $70 \% \%$ yield, a mixture of cis and trans isomers (d.r. 1.3: 1.0), which were partially separated by silica gel column chromatography (50\% EtOAc/Hexanes). 


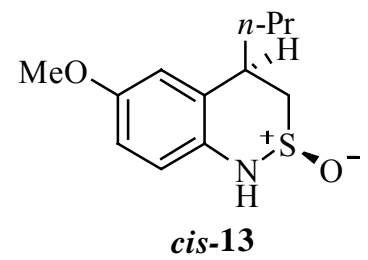

$1^{\text {st }}$ fraction, major isomer (cis): pale yellow solid, mp: $170-1{ }^{\circ} \mathrm{C}$; IR: $v 3154.1,2949.8$, 1033.3, $808.6 \mathrm{~cm}^{-1}$; ${ }^{1} \mathrm{H}-\mathrm{NMR}\left(500 \mathrm{MHz}, \mathrm{CDCl}_{3}\right): 6.78(\mathrm{~s}, 1 \mathrm{H}), 6.74(\mathrm{~s}, 1 \mathrm{H}), 6.73(\mathrm{~s}, 1 \mathrm{H})$, 6.50 (s, 1H), 3.79 (s, 3H), 3.29 (dd, $J=13.5,5.5 \mathrm{~Hz}, 1 \mathrm{H}$ ), 2.91-2.94 (m, 1H), 2.77 (ddd, $J$ $=14.0,7.5,1.5 \mathrm{~Hz}, 1 \mathrm{H}), 2.02-2.05(\mathrm{~m}, 1 \mathrm{H}), 1.91-1.95(\mathrm{~m}, 1 \mathrm{H}), 1.59-1.61(\mathrm{~m}, 1 \mathrm{H}), 1.46-$ $1.48(\mathrm{~m}, 1 \mathrm{H}), 1.00(\mathrm{t}, J=7.5 \mathrm{~Hz}, 3 \mathrm{H}) ;{ }^{13} \mathrm{C}-\mathrm{NMR}\left(125 \mathrm{MHz}, \mathrm{CDCl}_{3}\right): \delta 155.5,130.3$, 128.3，119.7, 113.6, 112.5, 52.5, 52.6, 35.5, 33.9, 20.7, 13.9; HRMS caclcd for $\mathrm{C}_{12} \mathrm{H}_{17} \mathrm{NO}_{2} \mathrm{SNa}^{+}[\mathrm{M}+\mathrm{Na}]^{+} 262.0872$, found 262.0868 .

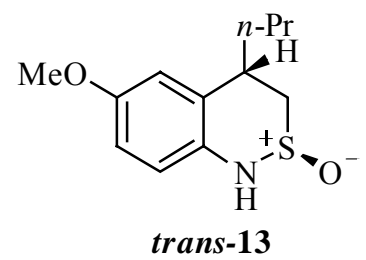

$2^{\text {nd }}$ fraction, minor isomer (trans): white solid, mp: $110-1{ }^{\circ} \mathrm{C}$; IR: $v$ 3170.4, 2837.5, 1466.5, 1217.2, 1045.6, $816.8 \mathrm{~cm}^{-1} ;{ }^{1} \mathrm{H}-\mathrm{NMR}\left(500 \mathrm{MHz}, \mathrm{CDCl}_{3}\right): 6.88$ (d, $\left.J=2.5 \mathrm{~Hz}, 1 \mathrm{H}\right), 6.68$ (dd, $J=8.5,1.5 \mathrm{~Hz}, 1 \mathrm{H}), 6.59$ (d, $J=8.5 \mathrm{~Hz}, 1 \mathrm{H}), 6.47$ (s, $1 \mathrm{H}), 3.77$ (s, 3H), 3.58-3.62 (m, 1H), 3.02 (ddd, $J=12.5,4.0,2.0 \mathrm{~Hz}, 1 \mathrm{H}), 2.67(\mathrm{t}, J=13.0 \mathrm{~Hz}, 1 \mathrm{H}), 2.01-2.06(\mathrm{~m}$, $1 \mathrm{H}), 1.82-1.84(\mathrm{~m}, 1 \mathrm{H}), 1.45-1.49$ (m, $1 \mathrm{H}), 1.34-1.38(\mathrm{~m}, 1 \mathrm{H}), 1.02$ (t, $J=7.5 \mathrm{~Hz}, 3 \mathrm{H})$; ${ }^{13} \mathrm{C}-\mathrm{NMR}\left(125 \mathrm{MHz}, \mathrm{CDCl}_{3}\right): \delta 154.9,128.7,127.4,118.4,112.9,112.5,55.6,49.4,34.1$, 26.2, 19.1, 14.2; HRMS caclcd for $\mathrm{C}_{12} \mathrm{H}_{17} \mathrm{NO}_{2} \mathrm{SNa}^{+}[\mathrm{M}+\mathrm{Na}]^{+} 262.0872$, found 262.0868 .

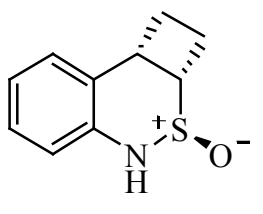

15

15: white solid, $59 \%$ yield (73\% yield based on recovered starting material), silica gel column chromatography (50\% EtOAc/Hexanes); mp: $220{ }^{\circ} \mathrm{C}$ (decomposed); IR: $v 3252.2$, 
1462.4, 1049.7, 747.3 $\mathrm{cm}^{-1}$; ${ }^{1} \mathrm{H}-\mathrm{NMR}$ (250 MHz, $\mathrm{CDCl}_{3}$ ): 7.13-7.19 (m, 1H), 6.99-7.08 (m, 2H), $6.79(\mathrm{~d}, J=7.4 \mathrm{~Hz}, 1 \mathrm{H}), 6.65(\mathrm{~s}, 1 \mathrm{H}), 4.00-4.09(\mathrm{~m}, 1 \mathrm{H}), 3.86-3.97(\mathrm{~m}, 1 \mathrm{H})$, 2.59-2.74 (m, 1H), 2.33-2.48 (m, 1H), 2.10-2.25 (m, 1H), 1.93-2.06 (m, 1H); ${ }^{13} \mathrm{C}-\mathrm{NMR}$ $\left(62.5 \mathrm{MHz}, \mathrm{CDCl}_{3}\right): \delta 132.8,129.7,128.6,127.8,123.8,120.6,57.7,34.2,28.8,20.2$; HRMS caclcd for $\mathrm{C}_{10} \mathrm{H}_{11} \mathrm{NOSNa}^{+}[\mathrm{M}+\mathrm{Na}]^{+} 216.0453$, found 216.0450; $[\alpha]^{25}{ }_{\mathrm{D}}=-262.1(\mathrm{c}$ $0.47, \mathrm{MeOH})$.

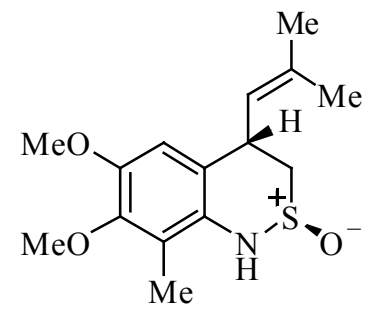

17

17: pale yellow liquid, $90 \%$ yield, silica gel column chromatography $(50 \%$ EtOAc/Hexanes); IR: v 3264.4, 2929.4, 1437.9, 1086.4, $874.0 \mathrm{~cm}^{-1} ;{ }^{1} \mathrm{H}-\mathrm{NMR}(500 \mathrm{MHz}$, $\left.\mathrm{CDCl}_{3}\right): 6.64(\mathrm{~s}, 1 \mathrm{H}), 6.30(\mathrm{~s}, 1 \mathrm{H}), 5.22(\mathrm{dt}, J=9.5,1.0 \mathrm{~Hz}, 1 \mathrm{H}), 4.48(\mathrm{ddd}, J=13.5,9.5$, $4.0 \mathrm{~Hz}, 1 \mathrm{H}), 3.79(\mathrm{~s}, 3 \mathrm{H}), 3.73(\mathrm{~s}, 3 \mathrm{H}), 2.89(\mathrm{ddd}, J=12.5,4.5,2.0 \mathrm{~Hz}, 1 \mathrm{H}), 2.62(\mathrm{t}, J=$ $13.0 \mathrm{~Hz}, 3 \mathrm{H}), 2.10(\mathrm{~s}, 3 \mathrm{H}), 1.86(\mathrm{~d}, J=1.0 \mathrm{~Hz}, 3 \mathrm{H}), 1.81(\mathrm{~d}, J=1.5 \mathrm{~Hz}, 3 \mathrm{H}) ;{ }^{13} \mathrm{C}-\mathrm{NMR}$ $\left(125 \mathrm{MHz}, \mathrm{CDCl}_{3}\right): \delta 147.5,146.6,136.2,127.1,123.7,120.2,118.4,110.6,60.5,56.2$, 48.5, 25.9, 25.8, 18.1, 9.5; HRMS caclcd for $\mathrm{C}_{15} \mathrm{H}_{21} \mathrm{NO}_{3} \mathrm{SNa}^{+}[\mathrm{M}+\mathrm{Na}]^{+} 318.1134$, found $318.1129 ;[\alpha]^{25}=+136.9\left(c 1.83, \mathrm{CHCl}_{3}\right)$.

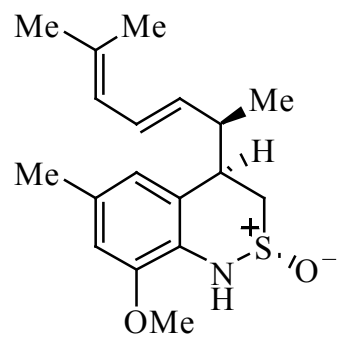

19

19: pale brown needle, $84 \%$ yield, silica gel column chromatography $(50 \%$ EtOAc/Hexanes); mp: $118-9{ }^{\circ} \mathrm{C}$; IR: v 3244.0, 2966.1, 2913.0, 1593.1, 1482.8, 1454.2, 
$1074.2 \mathrm{~cm}^{-1} ;{ }^{1} \mathrm{H}-\mathrm{NMR}\left(500 \mathrm{MHz}, \mathrm{CDCl}_{3}\right): 6.82(\mathrm{~s}, 1 \mathrm{H}), 6.81(\mathrm{~s}, 1 \mathrm{H}), 6.57(\mathrm{~s}, 1 \mathrm{H}), 6.26$ (ddd, $J=15.5,11.0,1.5 \mathrm{~Hz}, 1 \mathrm{H}), 5.71$ (d, $J=10.5 \mathrm{~Hz}, 1 \mathrm{H}), 5.43$ (dd, $J=15.0,5.5 \mathrm{~Hz}$, $1 \mathrm{H}), 3.83(\mathrm{~s}, 3 \mathrm{H}), 3.50(\mathrm{dt}, J=12.0,5.0 \mathrm{~Hz}, 1 \mathrm{H}), 3.37$ (q, $J=5.5 \mathrm{~Hz}, 1 \mathrm{H}), 2.91(\mathrm{ddd}, J=$ 13.0, 5.0, $2.5 \mathrm{~Hz}, 1 \mathrm{H}), 2.71(\mathrm{t}, J=12.5 \mathrm{~Hz}, 1 \mathrm{H}), 1.73(\mathrm{~s}, 3 \mathrm{H}), 1.72(\mathrm{~s}, 3 \mathrm{H}), 1.21(\mathrm{~d}, J=$ $6.5 \mathrm{~Hz}, 3 \mathrm{H}) ;{ }^{13} \mathrm{C}-\mathrm{NMR}\left(125 \mathrm{MHz}, \mathrm{CDCl}_{3}\right): \delta 147.7,134.1,131.6,130.9,127.4,125.2$, $124.9,122.4,119.5,109.4,55.6,46.5,35.9,32.7,25.8,21.5,18.2,17.5$; HRMS caclcd for $\mathrm{C}_{18} \mathrm{H}_{25} \mathrm{NO}_{2} \mathrm{SNa}^{+}[\mathrm{M}+\mathrm{Na}]^{+} 342.1498$, found $342.1497 ;[\alpha]^{25}=+162.8$ (c 1.82, $\left.\mathrm{CHCl}_{3}\right)$.

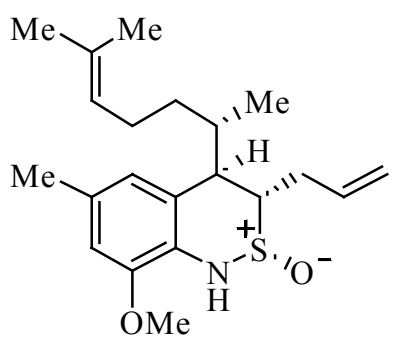

21

21: off-white needle, $89 \%$ yield (d.r. $8.2: 1.0)$, silica gel column chromatography ( $40 \%$ EtOAc/Hexanes); mp: 85-6 ${ }^{\circ} \mathrm{C}$; IR: v 3231.7, 2913.0, 1597.2, 1486.9, 1450.1, 1074.2, 833.1, $726.9 \mathrm{~cm}^{-1}$; ${ }^{1} \mathrm{H}-\mathrm{NMR}\left(500 \mathrm{MHz}, \mathrm{CDCl}_{3}\right): 6.68(\mathrm{~s}, 1 \mathrm{H}), 6.55(\mathrm{~s}, 1 \mathrm{H}), 6.54(\mathrm{~s}, 1 \mathrm{H})$, 5.88-5.94 (m, 1H), $5.24(\mathrm{dd}, J=17.0,1.0 \mathrm{~Hz}, 1 \mathrm{H}), 5.19(\mathrm{~d}, J=10.0 \mathrm{~Hz}, 1 \mathrm{H}), 5.03(\mathrm{t}, J=$ $7.0 \mathrm{~Hz}, 1 \mathrm{H}), 3.81(\mathrm{~s}, 3 \mathrm{H}), 2.93(\mathrm{dt}, J=9.5,5.5 \mathrm{~Hz}, 1 \mathrm{H}), 2.83(\mathrm{dd}, J=6.0,3.5 \mathrm{~Hz}, 1 \mathrm{H})$, $2.71(\mathrm{dt}, J=15.0,8.0 \mathrm{~Hz}, 1 \mathrm{H}), 2.34(\mathrm{td}, J=15.0,5.5 \mathrm{~Hz}, 1 \mathrm{H}), 2.28(\mathrm{~s}, 3 \mathrm{H}), 2.00-2.04(\mathrm{~m}$, $1 \mathrm{H}), 1.84-1.92(\mathrm{~m}, 2 \mathrm{H}), 1.68(\mathrm{~s}, 3 \mathrm{H}), 1.58(\mathrm{~s}, 3 \mathrm{H}), 1.37-1.40(\mathrm{~m}, 1 \mathrm{H}), 0.99-1.04(\mathrm{~m}, 1 \mathrm{H})$, $0.92(\mathrm{~d}, J=7.0 \mathrm{~Hz}, 3 \mathrm{H}) ;{ }^{13} \mathrm{C}-\mathrm{NMR}\left(125 \mathrm{MHz}, \mathrm{CDCl}_{3}\right): \delta 149.1,134.1,132.0,131.6$, $128.4,124.1,121.9,120.8,118.5,109.5,60.7,55.5,44.3,38.8,35.3,33.6,25.9,25.6$, 21.4, 17.6, 16.7; HRMS caclcd for $\mathrm{C}_{21} \mathrm{H}_{31} \mathrm{NO}_{2} \mathrm{SNa}^{+}[\mathrm{M}+\mathrm{Na}]^{+}$384.1967, found 384.1955. 


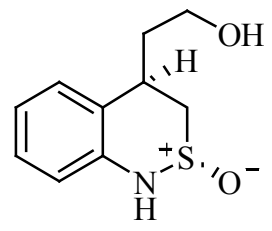

23

23: brown liquid, 70\% yield, silica gel column chromatography $(\mathrm{MeOH})$; IR: $v$ 3317.5, 1560.5, 1462.4, 1405.2, 1045.6, 898.5, $751.4 \mathrm{~cm}^{-1}$; ${ }^{1} \mathrm{H}-\mathrm{NMR}$ (500 MHz, $\left.\mathrm{d}_{4}-\mathrm{MeOD}\right): 7.36$ $(\mathrm{d}, J=7.5 \mathrm{~Hz}, 1 \mathrm{H}), 7.13(\mathrm{dd}, J=7.5,0.5 \mathrm{~Hz}, 1 \mathrm{H}), 6.96(\mathrm{dd}, J=7.5,1.0 \mathrm{~Hz}, 1 \mathrm{H}), 6.78$ $(\mathrm{dd}, J=8.0,1.5 \mathrm{~Hz}, 1 \mathrm{H}), 3.61-3.75(\mathrm{~m}, 3 \mathrm{H}), 3.30-3.31(\mathrm{~m}, 1 \mathrm{H}), 3.13(\mathrm{dd}, J=13.0,4.5$ $\mathrm{Hz}, 1 \mathrm{H}), 2.73$ (t, $J=13.0 \mathrm{~Hz}, 1 \mathrm{H}), 2.39-2.45(\mathrm{~m}, 1 \mathrm{H}), 1.98-2.04(\mathrm{~m}, 1 \mathrm{H}) ;{ }^{13} \mathrm{C}-\mathrm{NMR}(125$ $\left.\mathrm{MHz}, \mathrm{d}_{4}-\mathrm{MeOD}\right): \delta 136.9,128.5,127.6,126.8,122.9,118.5,60.1,49.6,35.6,24.8$; HRMS cacled for $\mathrm{C}_{10} \mathrm{H}_{13} \mathrm{NO}_{2} \mathrm{SNa}^{+}[\mathrm{M}+\mathrm{Na}]^{+} 234.0559$, found 234.0559.

\section{Quantitative GC Experiment}

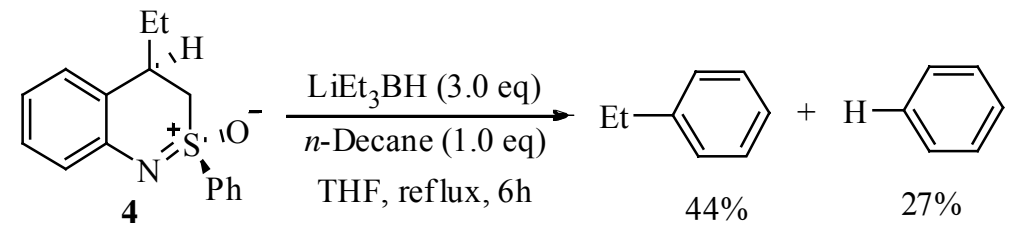

To the THF solution of 4 and $n$-decane at $0{ }^{\circ} \mathrm{C}$, was dropwisely added lithium triethylborohydride (1.0M in THF solution). The resulting mixture was refluxed for $6.0 \mathrm{~h}$. The reaction mixture was cooled to room temperature and quenched by the sequential addition of methanol, $1 \mathrm{~N} \mathrm{NaOH}$ and hydrogen peroxide solution. The reaction mixture was extracted with $\mathrm{CH}_{2} \mathrm{Cl}_{2}$ (3 times) and dry over $\mathrm{MgSO}_{4}$. The crude mixture was subjected to GC-MS analysis. GC conditions were as followed: flow rate: $1 \mathrm{ml} / \mathrm{min} ; 70{ }^{\circ} \mathrm{C}$, $5 \mathrm{~min}, 10{ }^{\circ} \mathrm{C} / \mathrm{min}, 200{ }^{\circ} \mathrm{C}, 1 \mathrm{~min}$. Ethyl benzene was obtained in $44 \%$ yield and benzene was obtained in $27 \%$ yield.

\section{$\underline{\text { Reference }}$}

${ }^{1}$ Harmata, M.; Hong, X. J. Am. Chem. Soc. 2003, 125, 5754.

${ }^{2}$ Harmata, M.; Jones, D. E. Tetrahedron Lett. 1993, 36, 4769.

${ }^{3}$ Harmata, M.; Kahraman, M. J. Org. Chem. 1998, 63, 6845.

${ }^{4}$ Harmata, M.; Hong, X.; Barnes, C. L. Org. Lett. 2004, 6, 2201. 


\section{Copies of}

\section{${ }^{1}$ H-NMR and ${ }^{13} \mathrm{C}$-NMR}



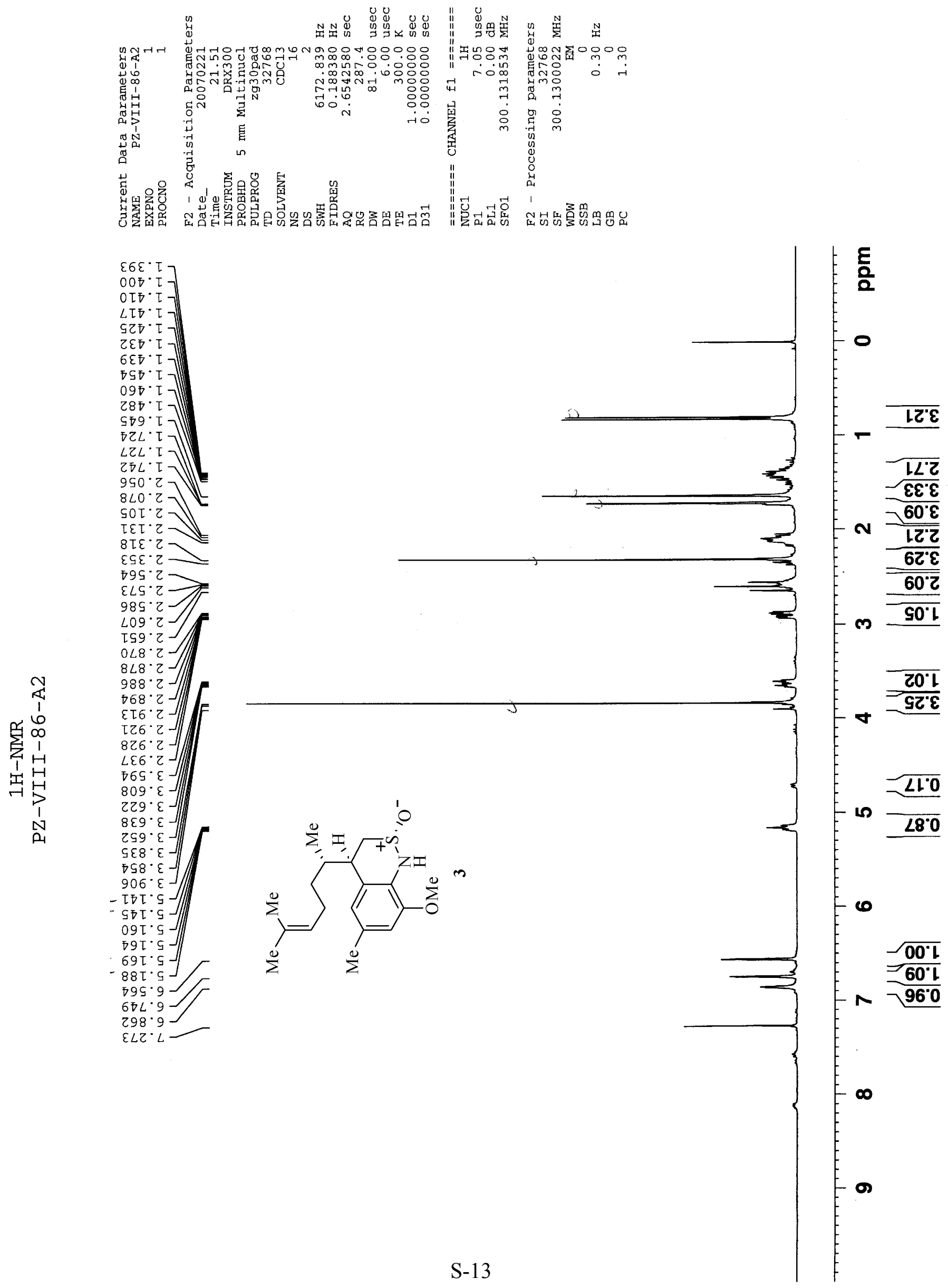

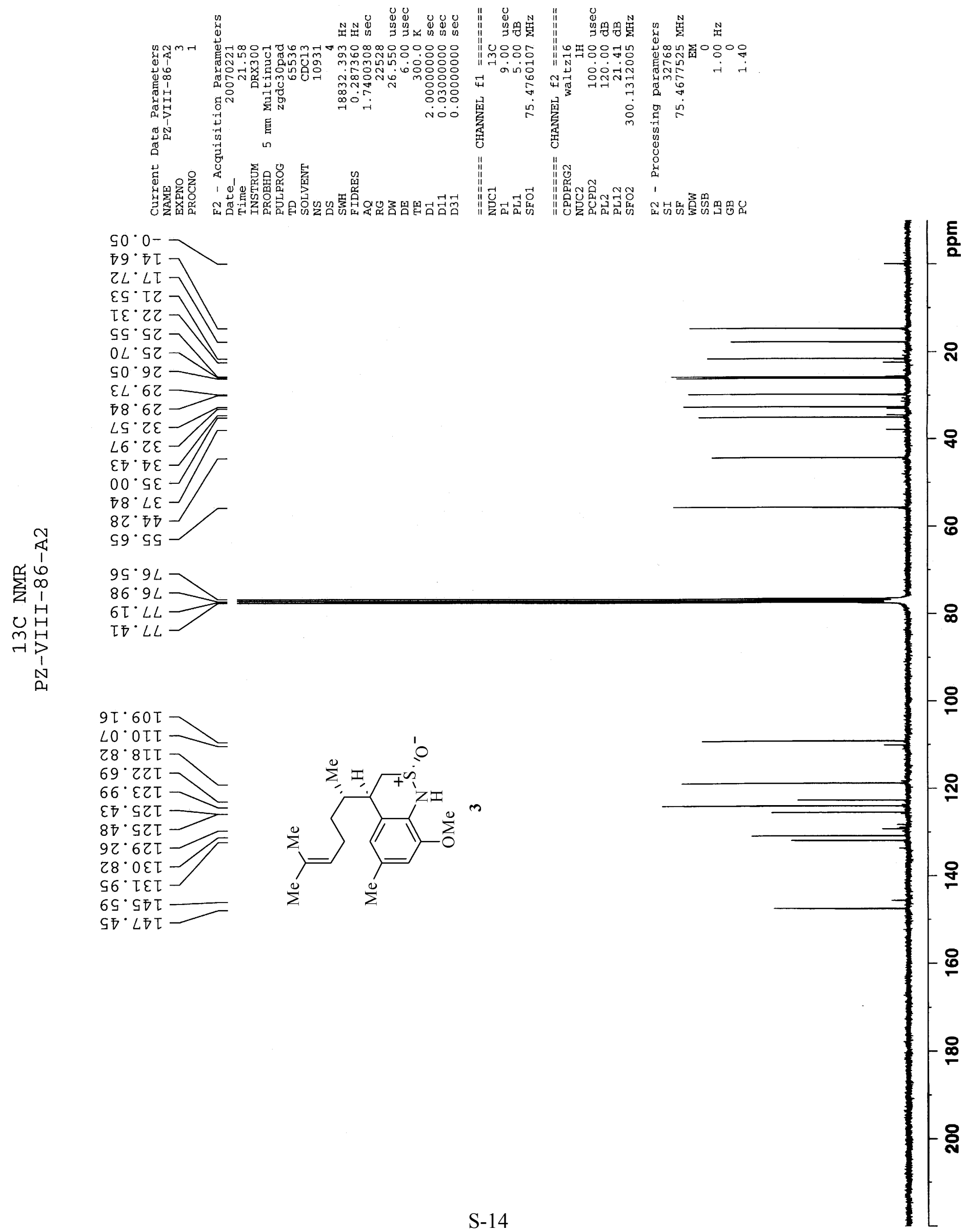


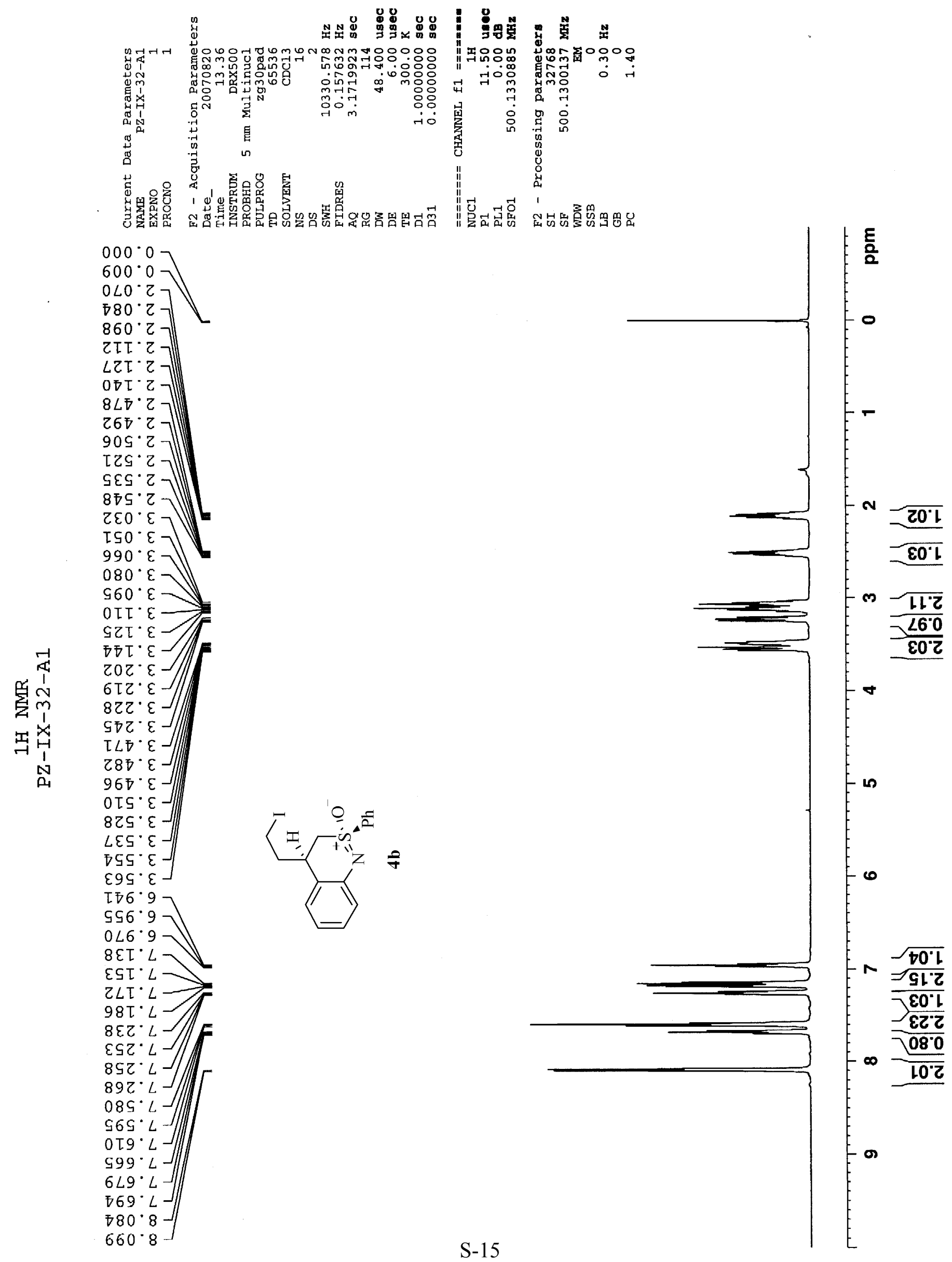




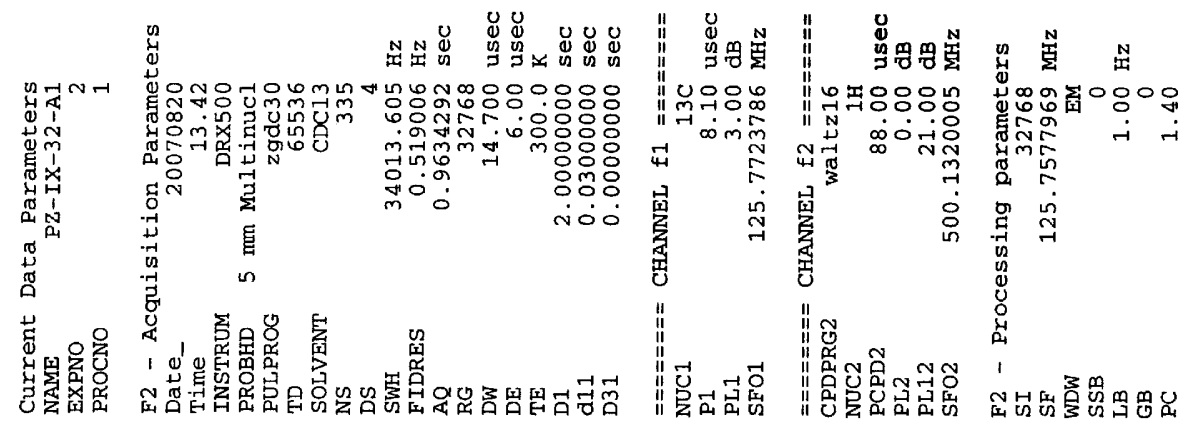

$80^{\circ} 0^{\circ-}=$

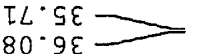

$2 S^{\cdot} \mathrm{ZS}$

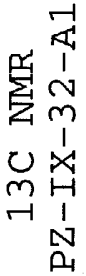
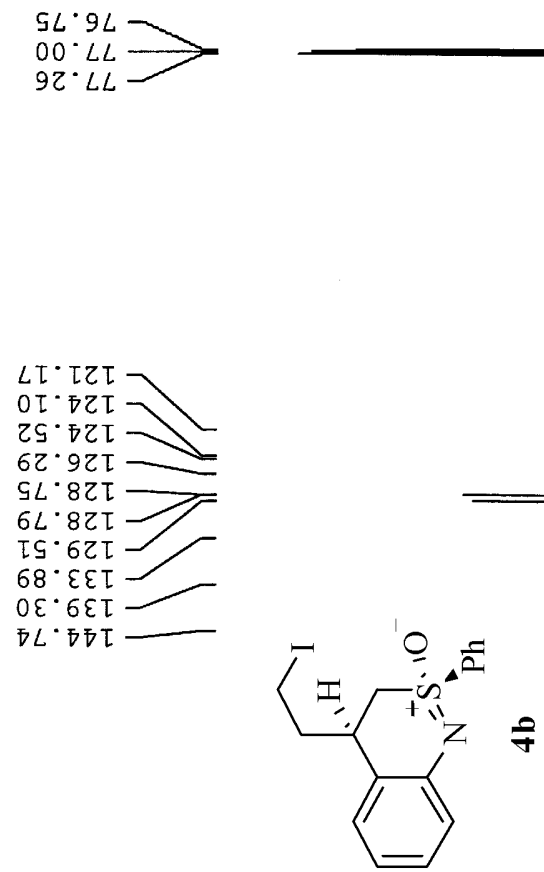


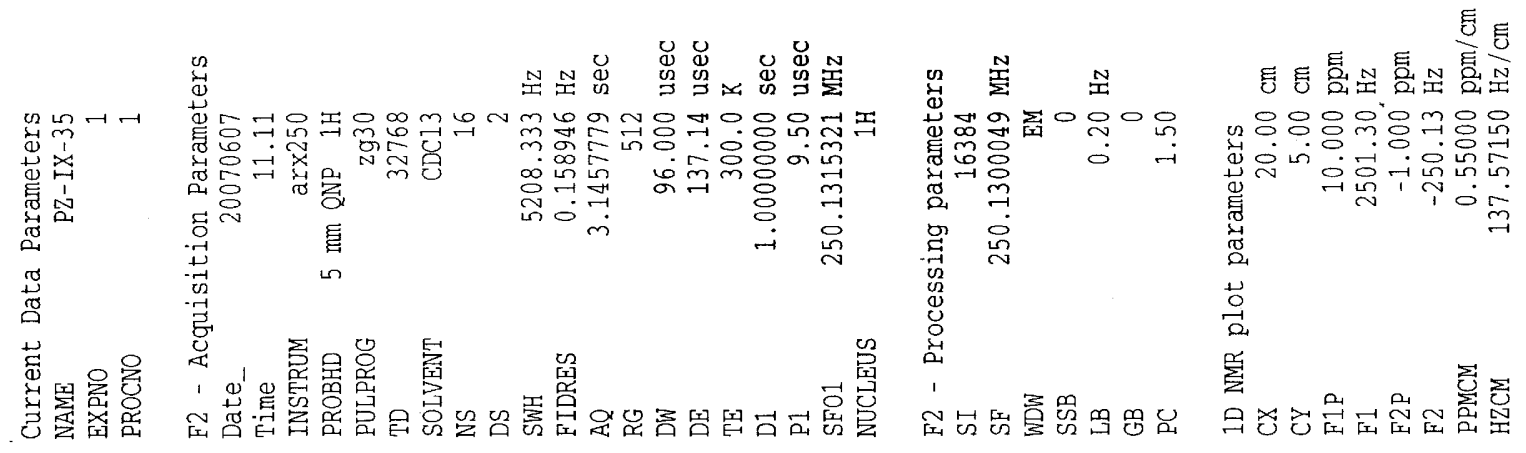

䚁点
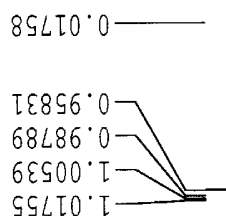

SSLTO

IELO

L๐G

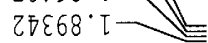

9ELZT'

T69EI' 2

ZILST'?

โ929T'

$27518^{\circ} 7$

$20026^{\circ} \mathrm{Z}$

$59996^{\circ} \mathrm{Z}$

$\angle 9900^{\circ} \varepsilon \longrightarrow$

$6 \varepsilon \varepsilon 2 \overline{6} \cdot \varepsilon$

$\angle 6 \angle 90^{\circ}$

$60660^{\circ}$

L9STS. $\varepsilon-$

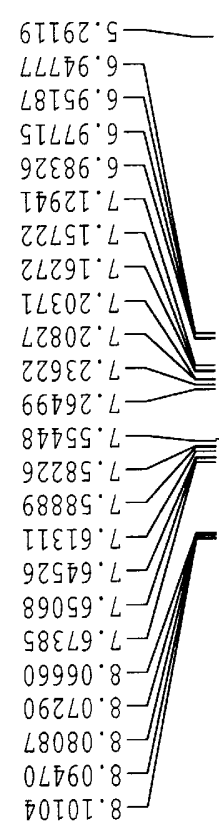

urdd

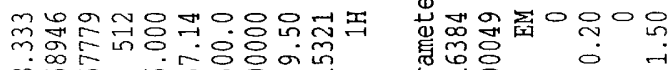

ฉ

总 윰

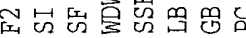




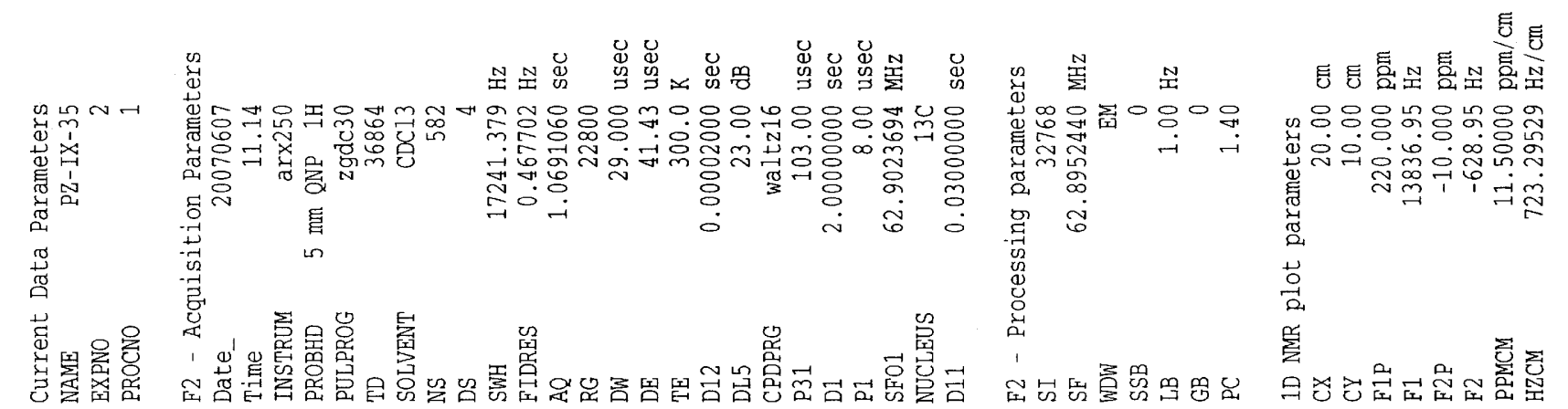

LOS'OT

万or'

$6 \angle 9^{\circ} \pi \varepsilon$

ธ9์' โG

$\sum_{\substack{n \\ m}}^{n}$
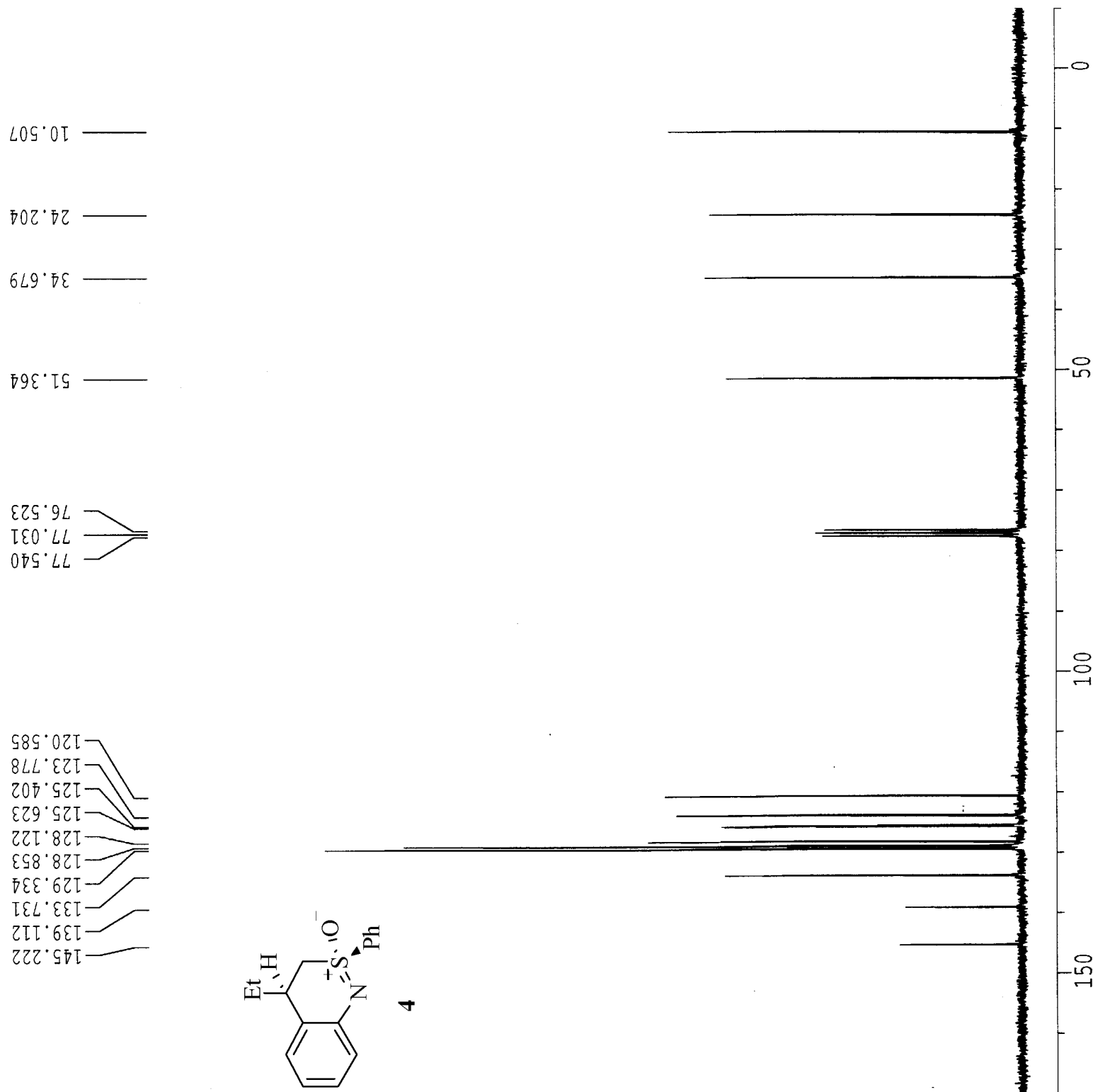


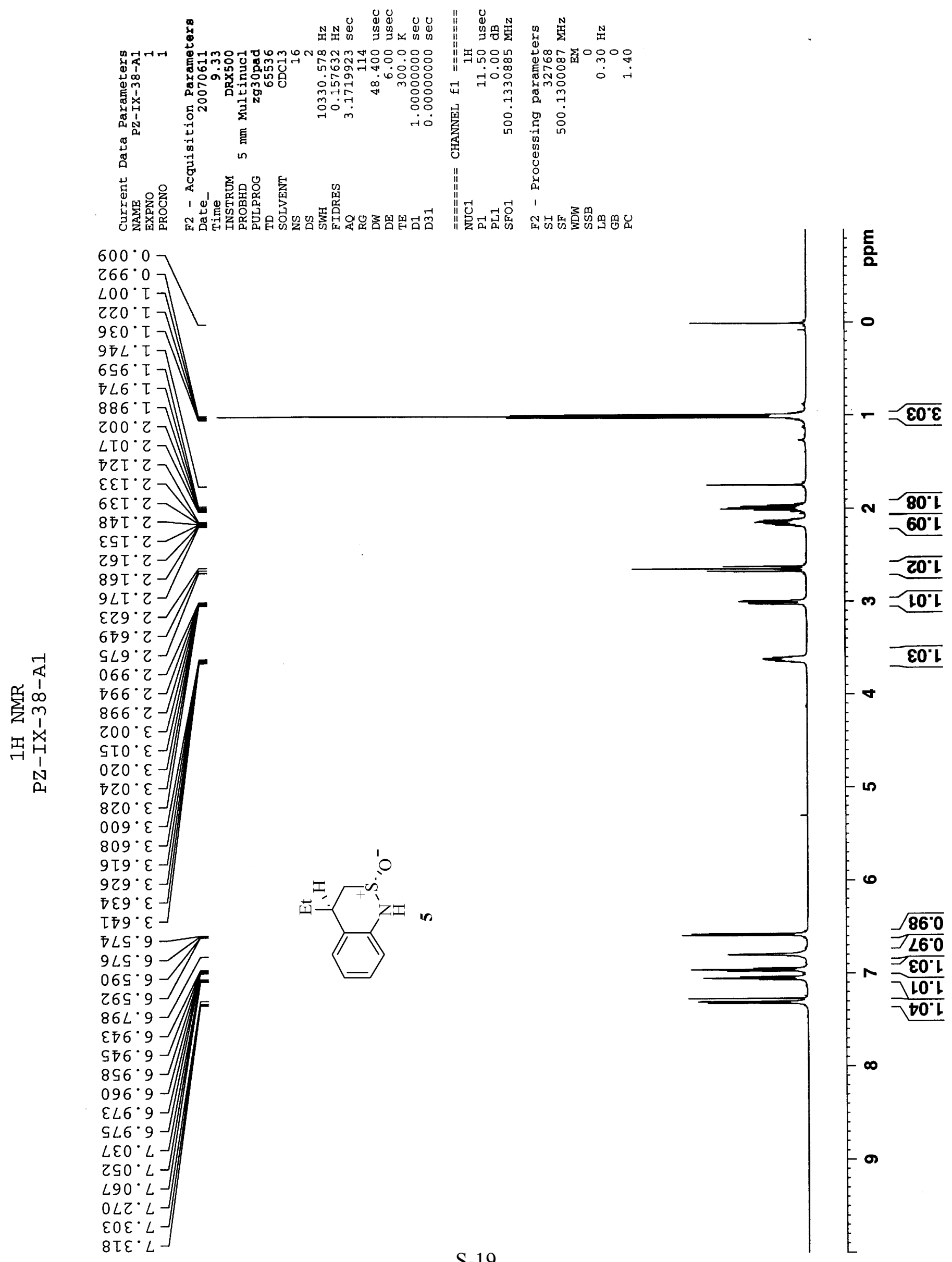



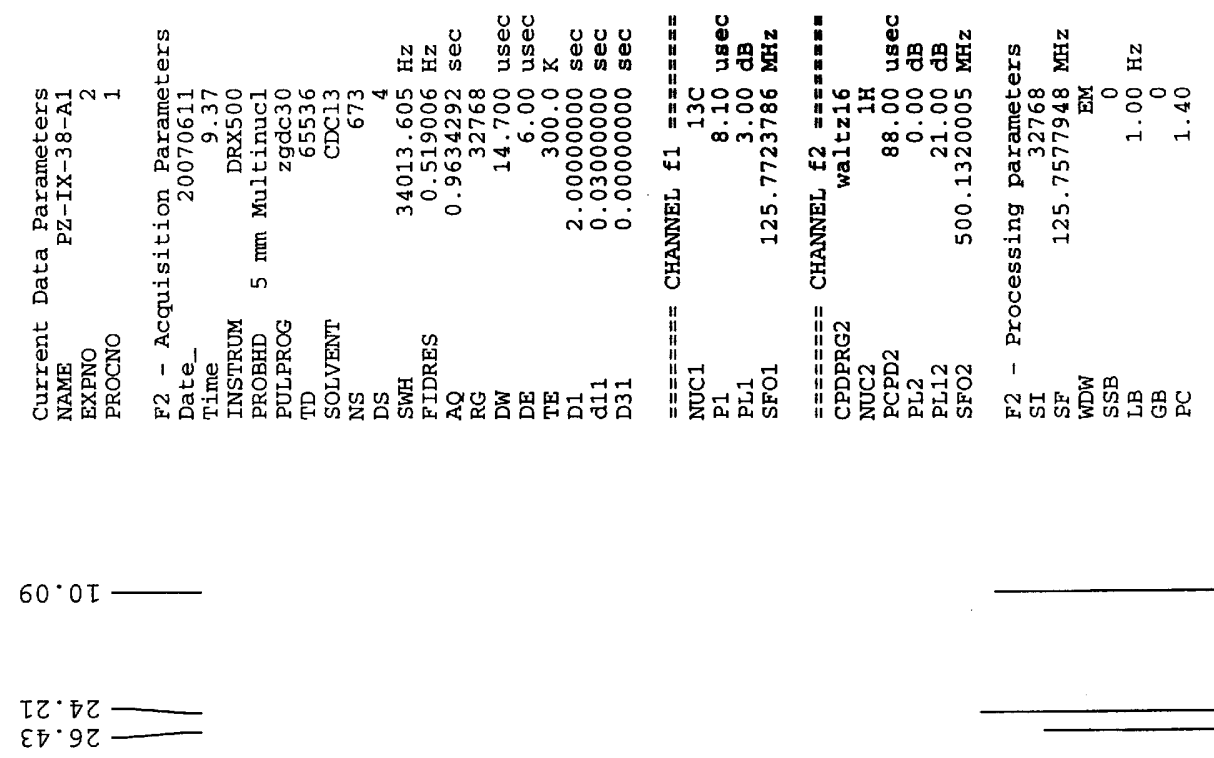

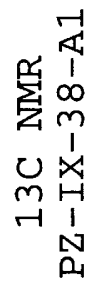

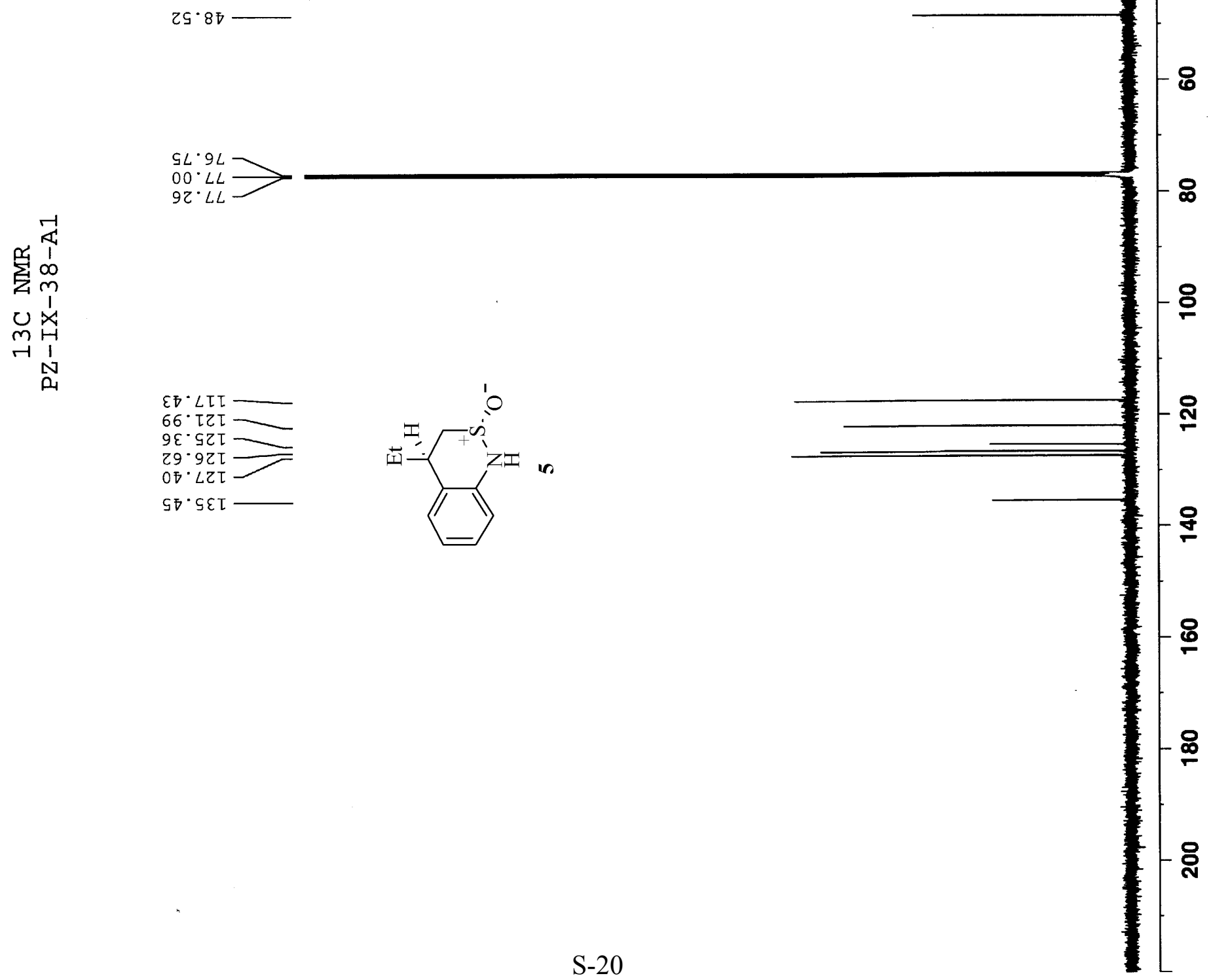




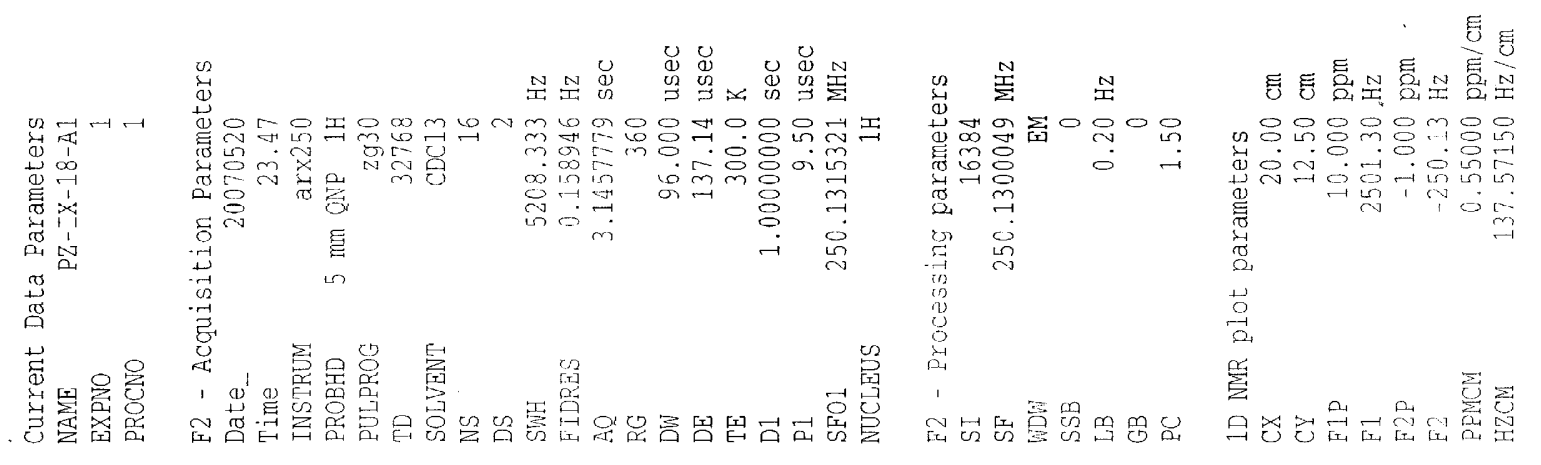

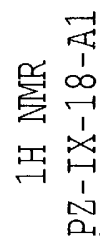
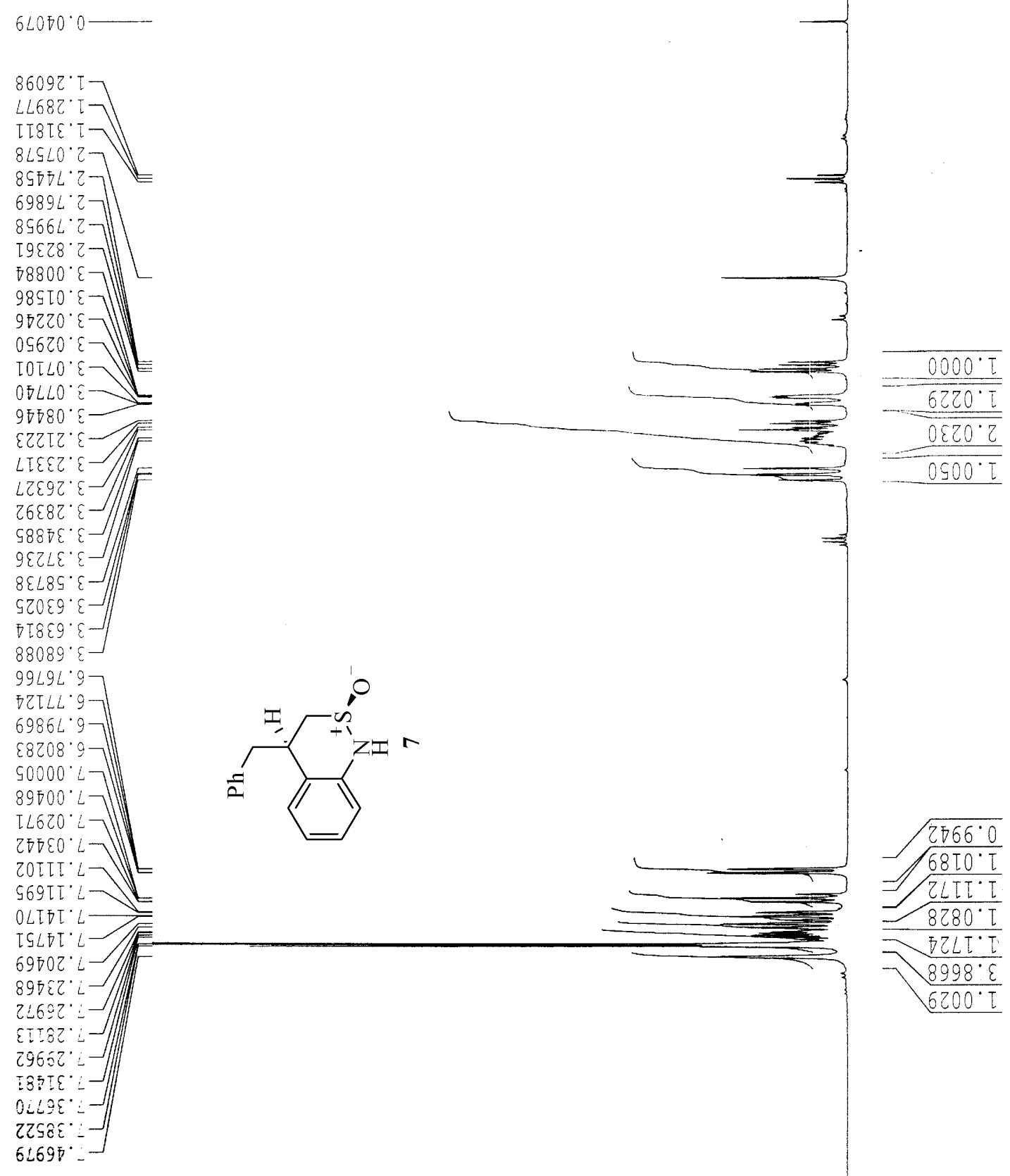


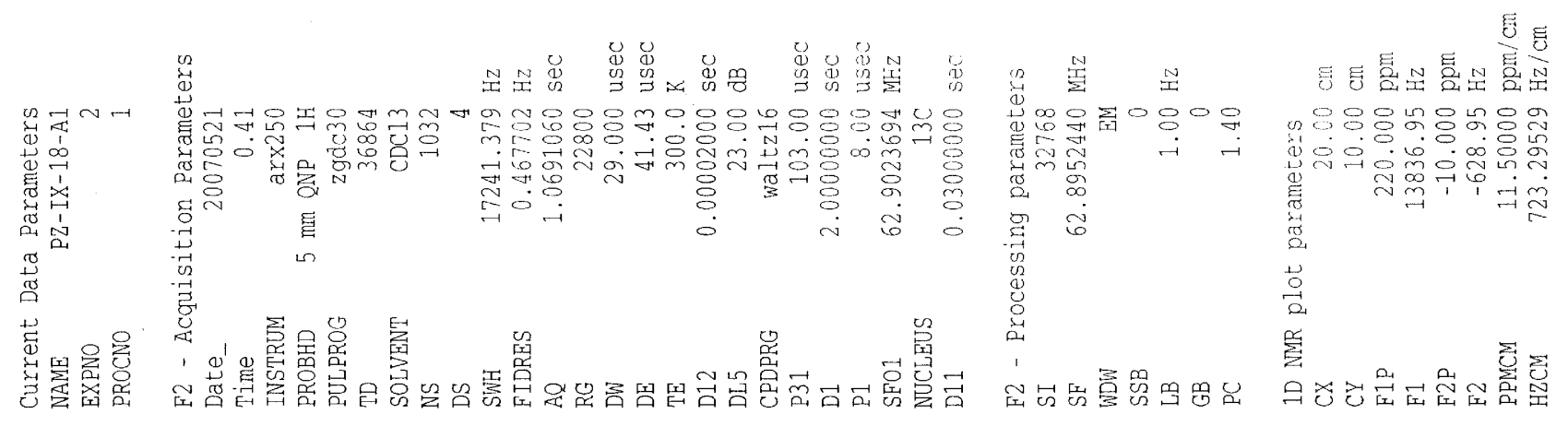

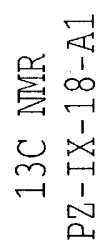
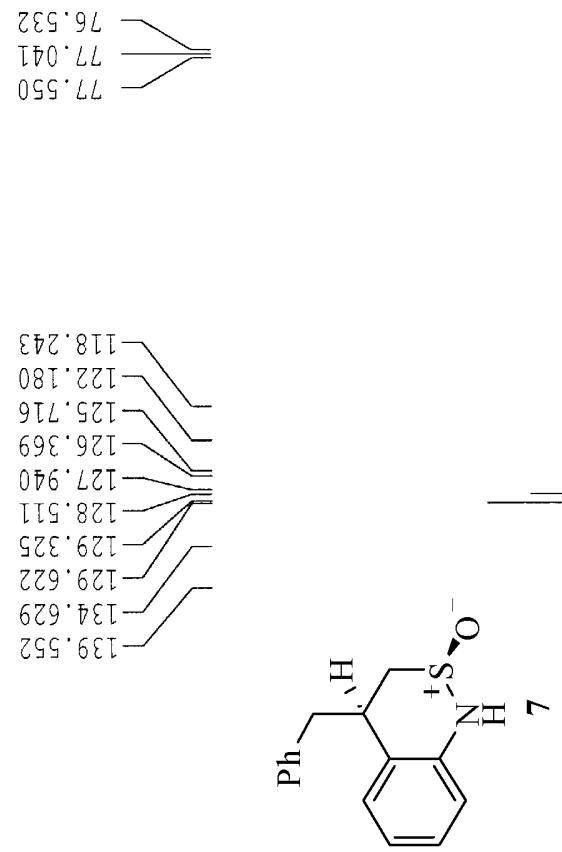


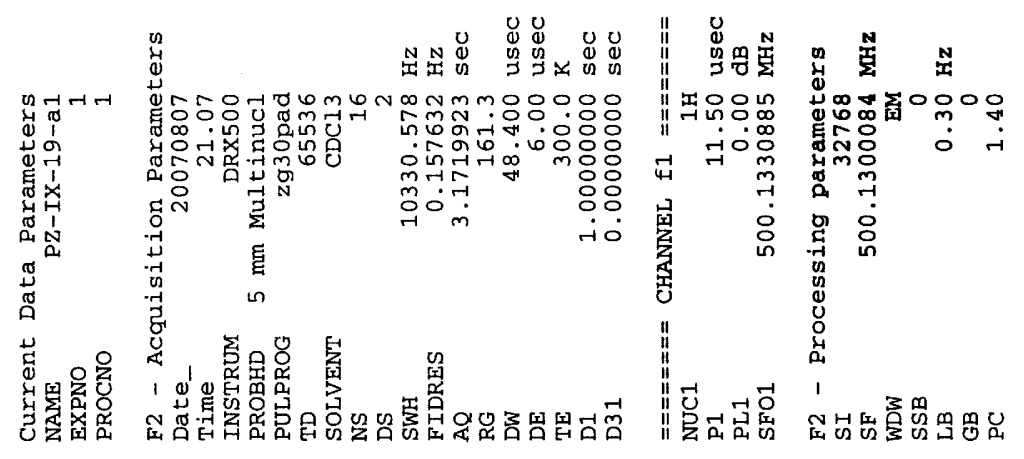

$600^{\circ} 0$

孚先
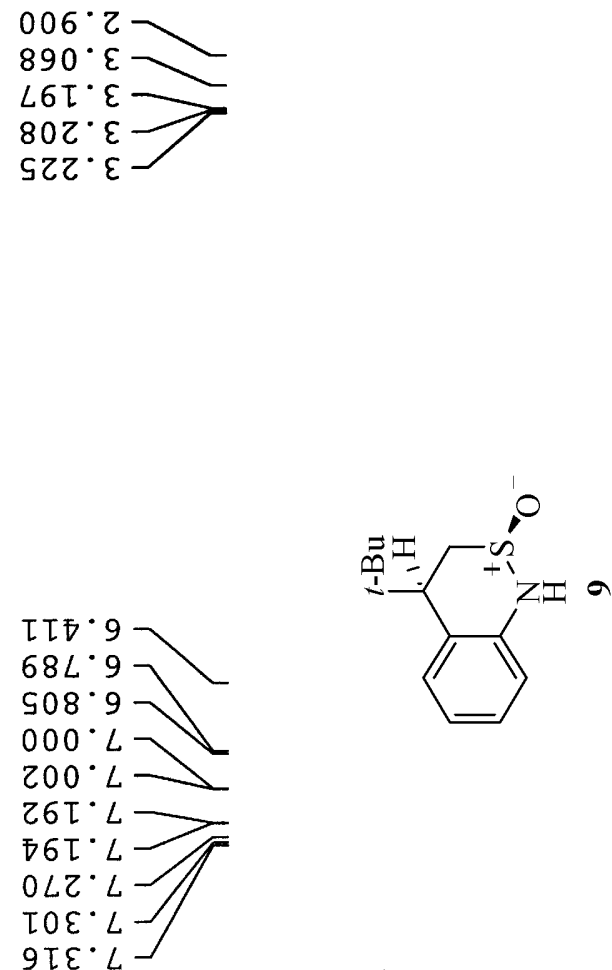

| $\sqrt{66^{\circ} 8}$ $\sqrt{80.1}$ $\frac{70.1}{70.1}$ 


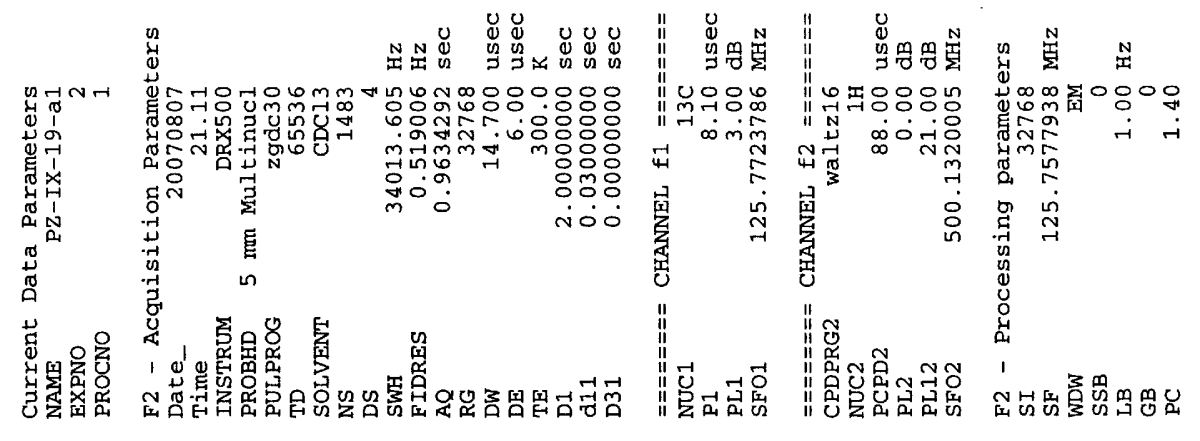

a
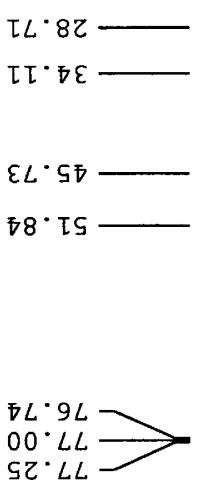

$\varepsilon L^{\cdot} \mathrm{S} \overline{ }$

$78^{\circ}$. TS

$$
\mathrm{GZ} \cdot L L
$$
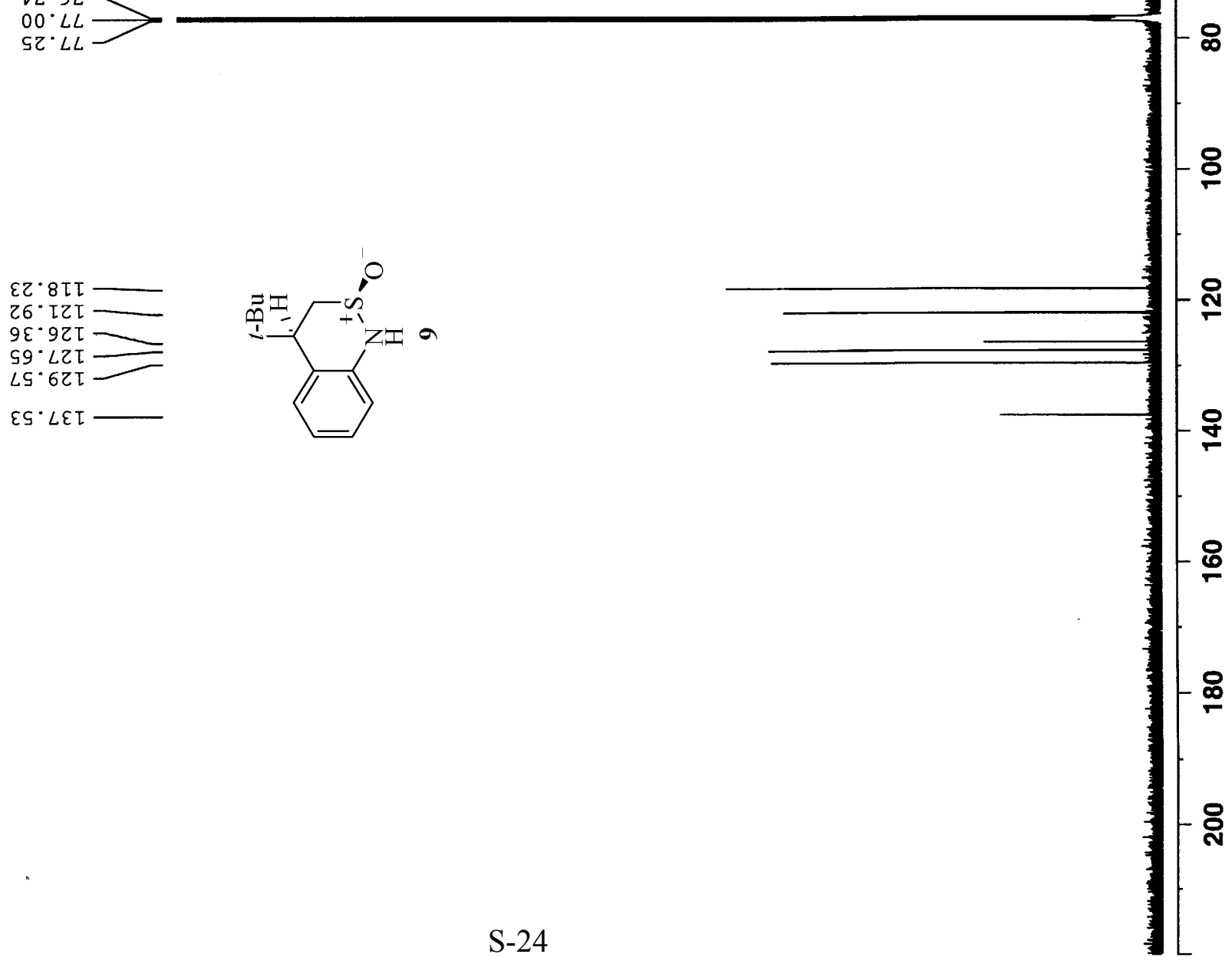


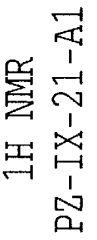
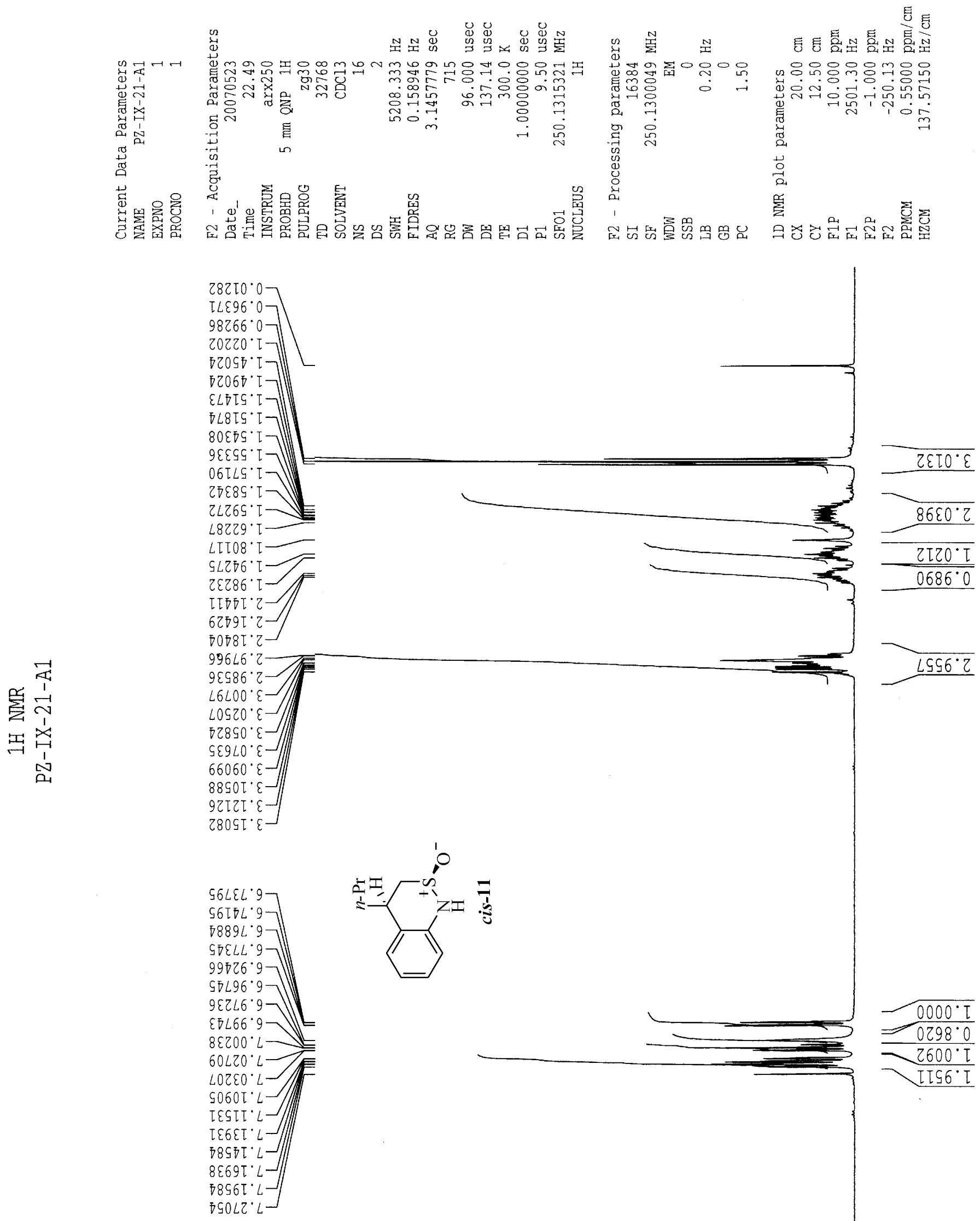

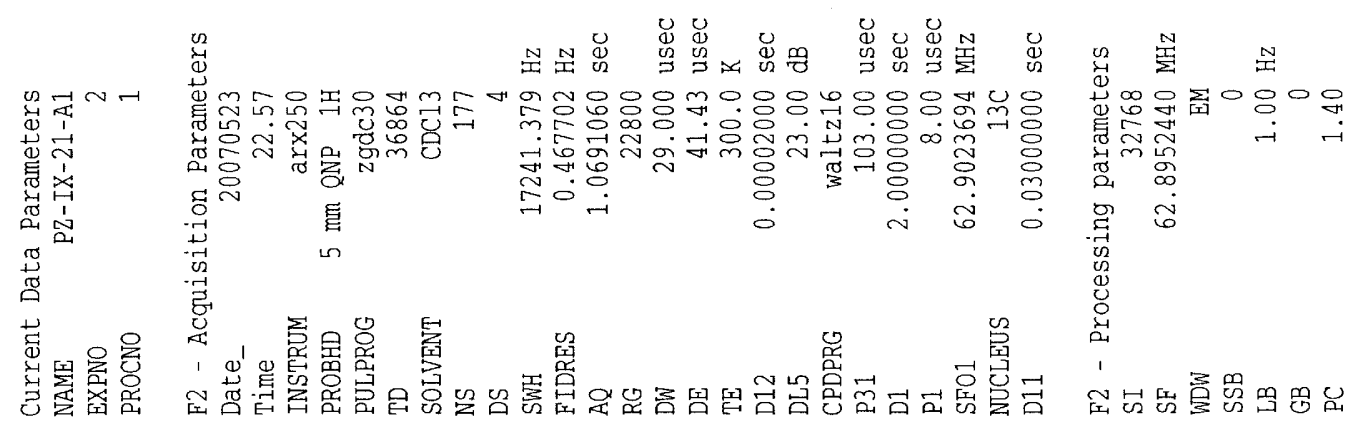

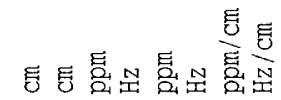

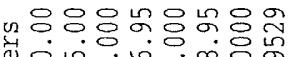

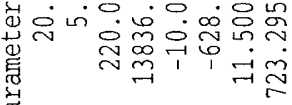
वี 蒙

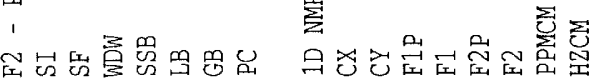
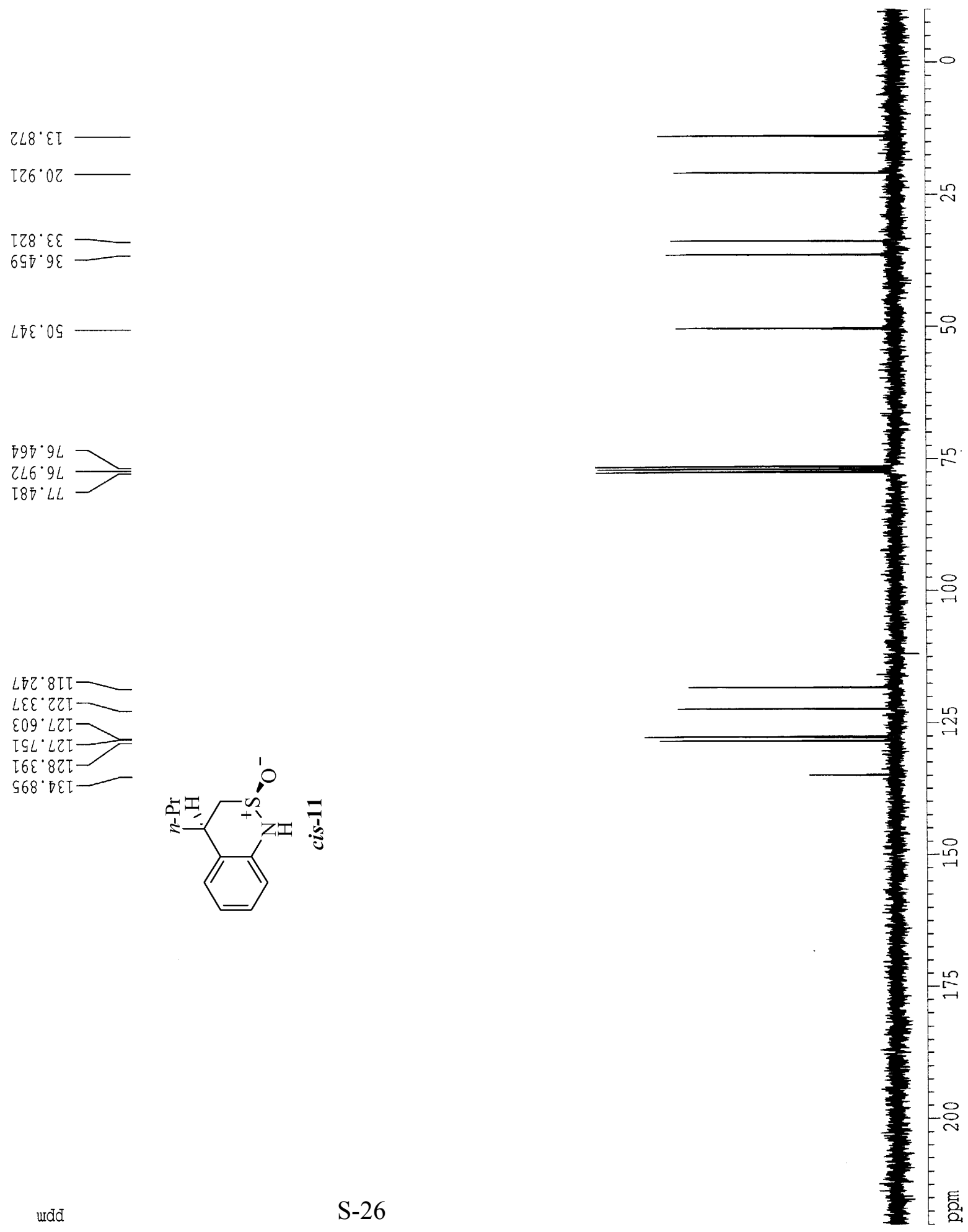


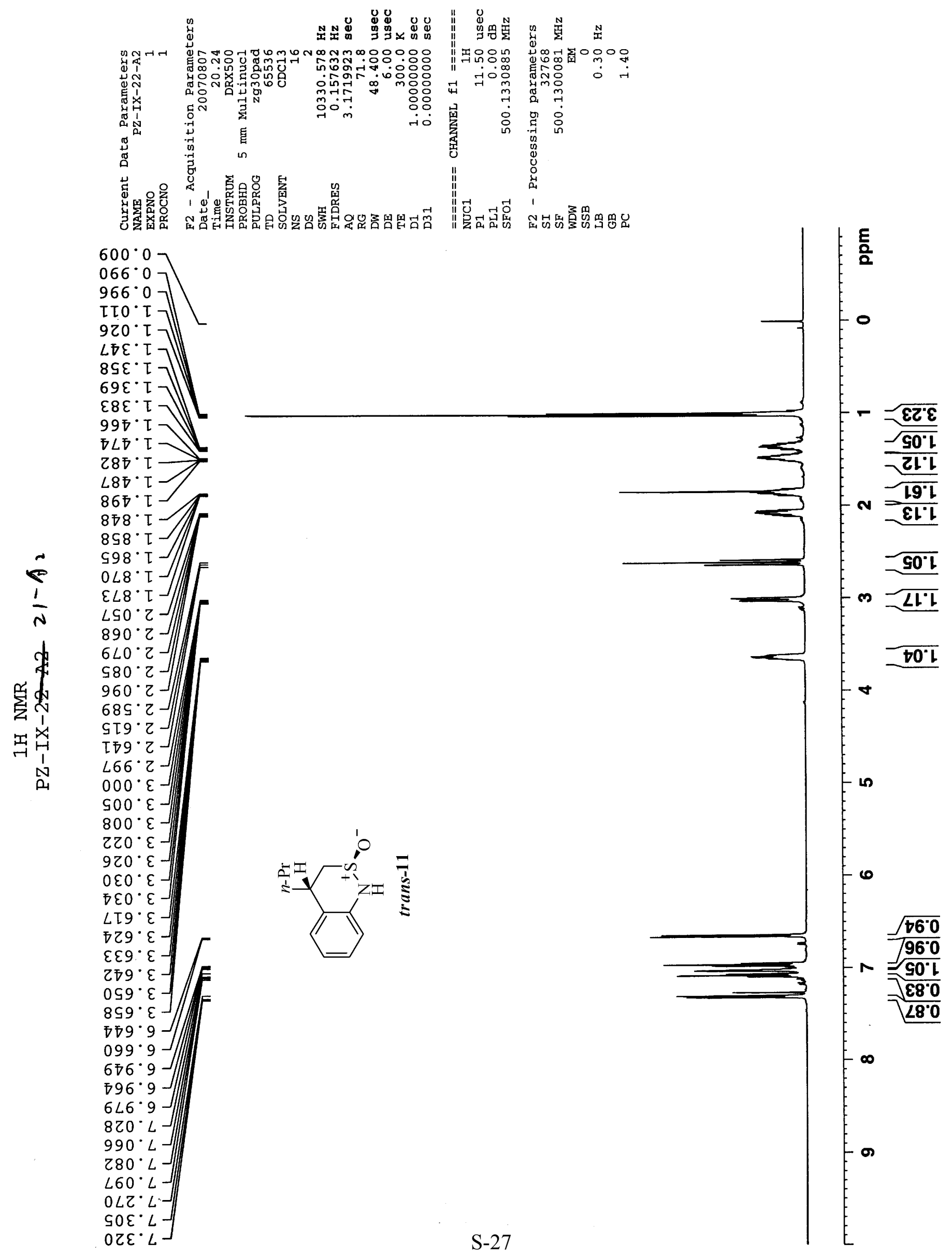



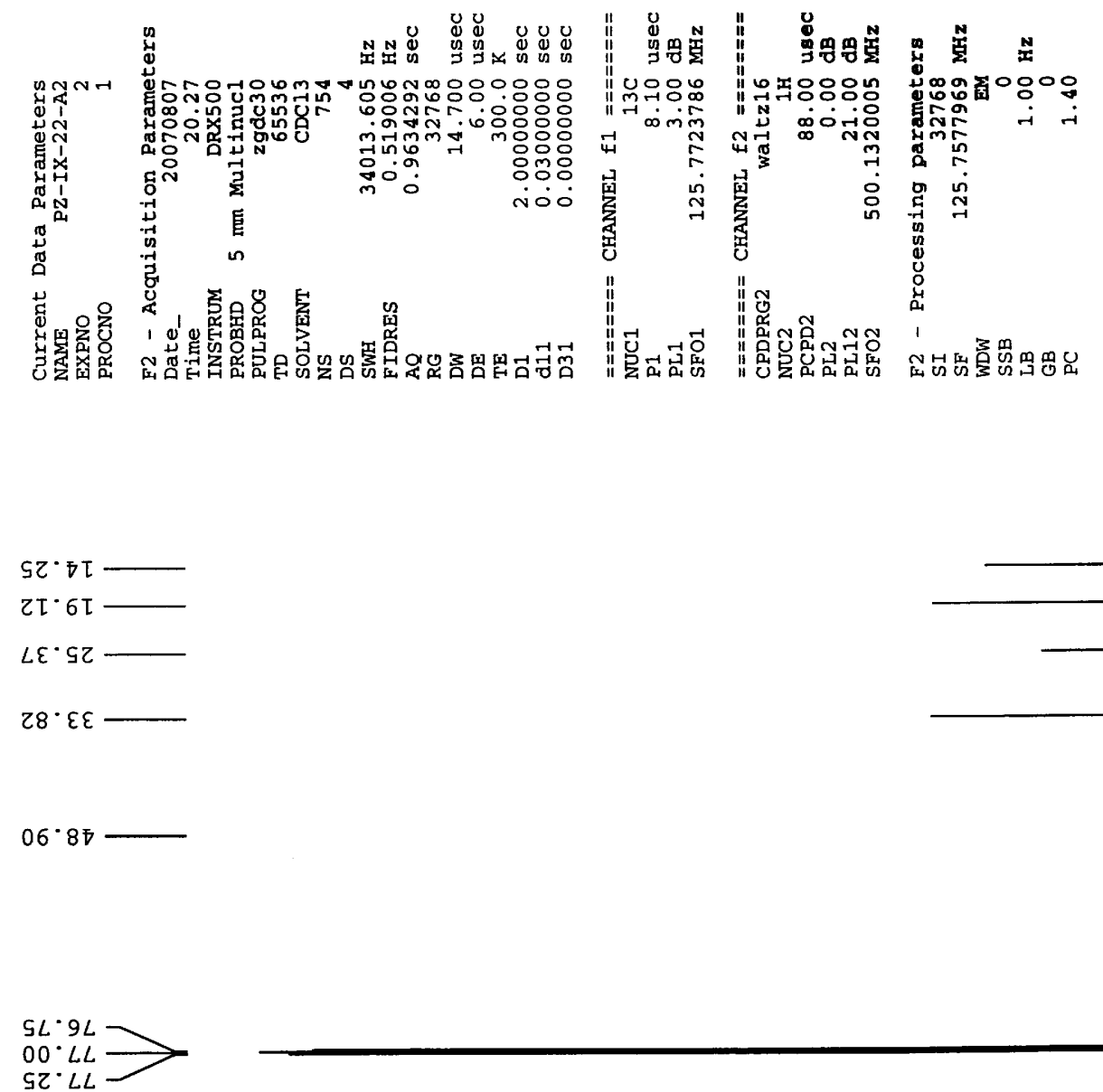

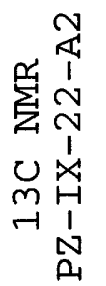
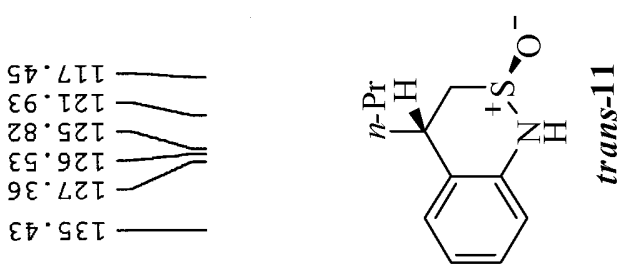


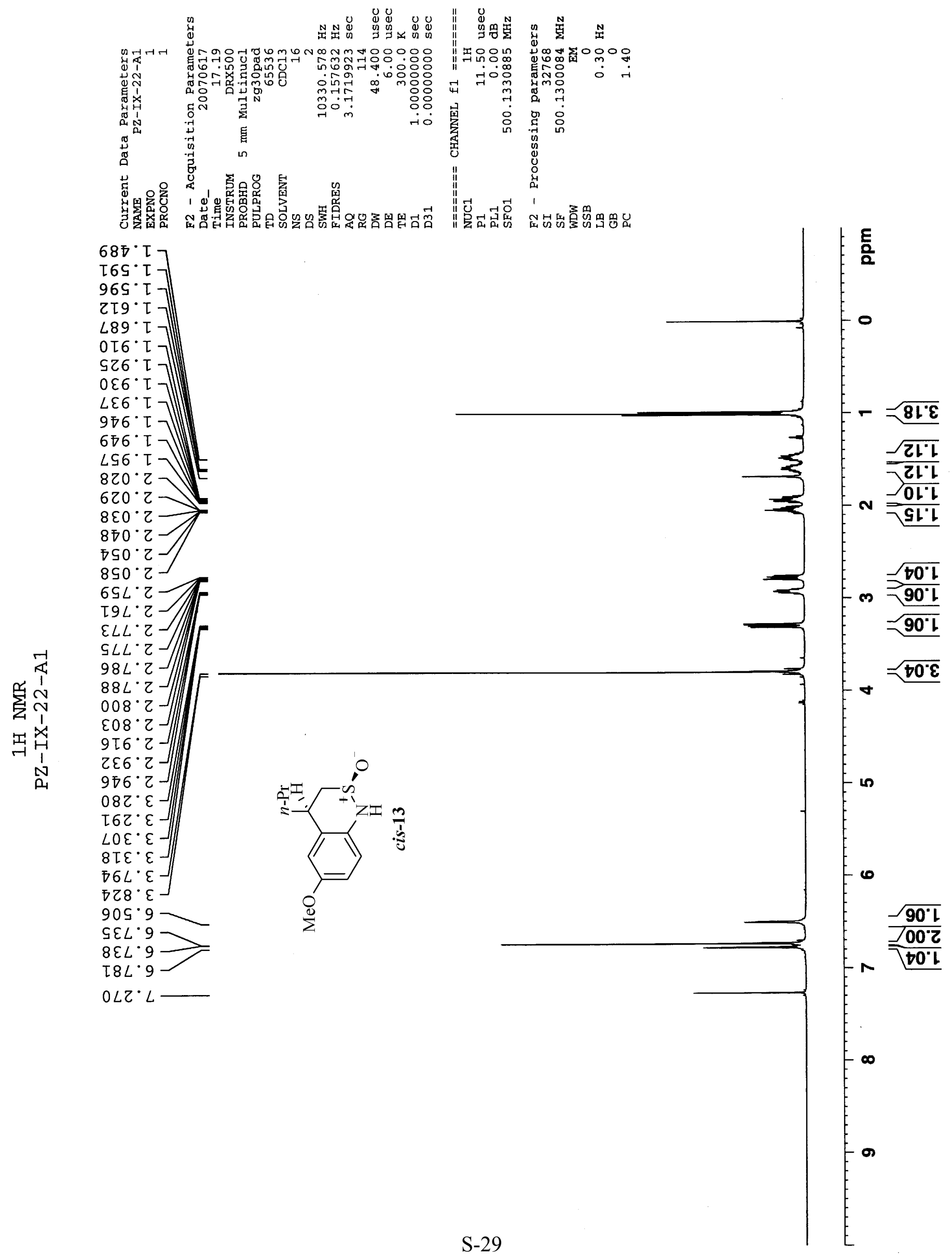



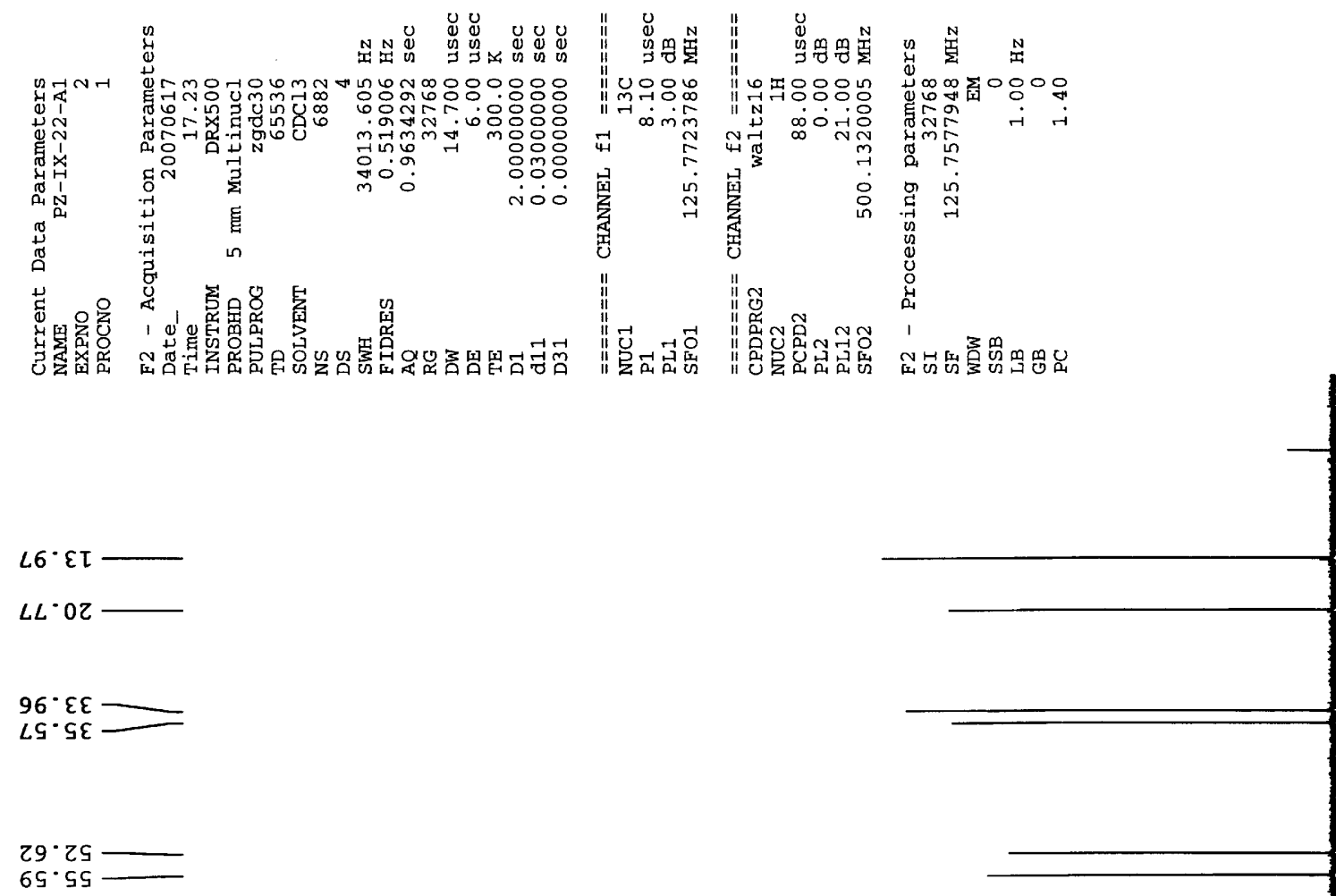

$29 \cdot 25=$
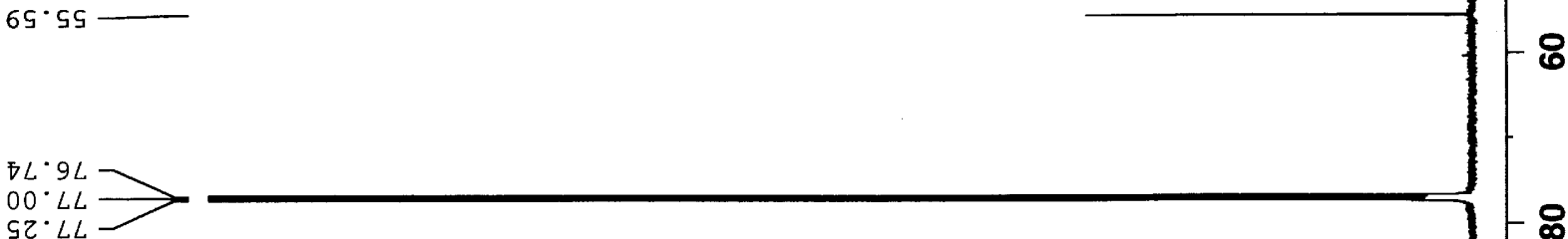

N

$$
00^{\circ} L L
$$
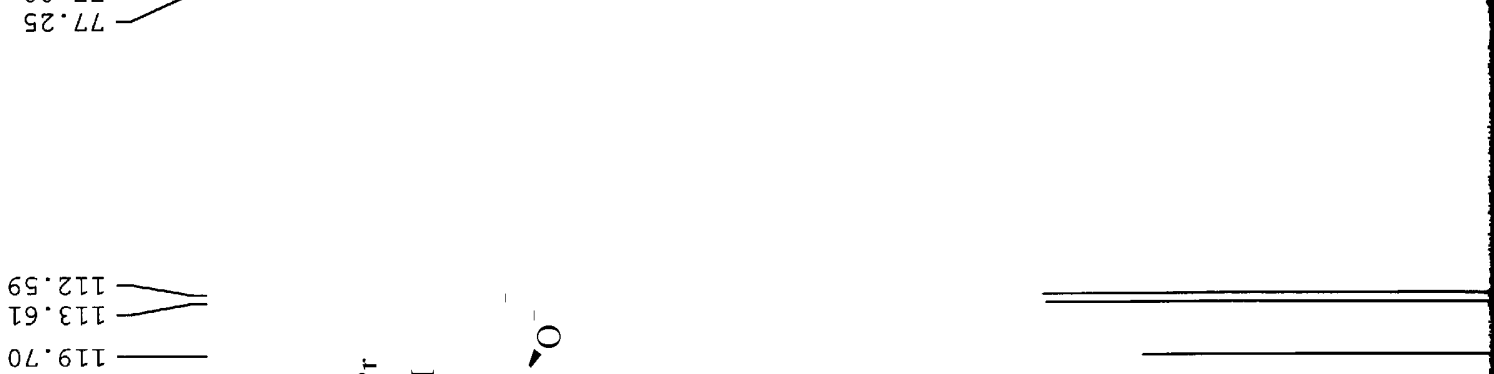

$Z \varepsilon \cdot 8 Z I$

$9 \varepsilon \cdot 0 \varepsilon \tau$

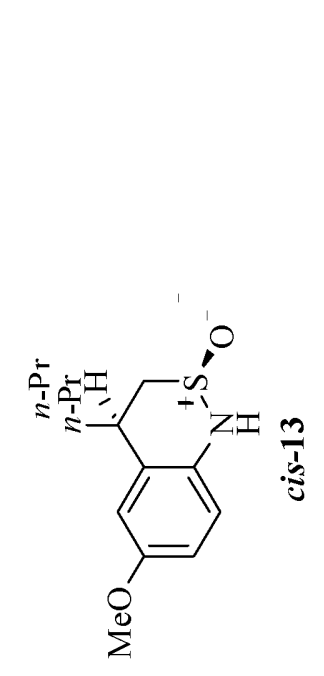

$0 S^{\cdot S S T ~}$ 

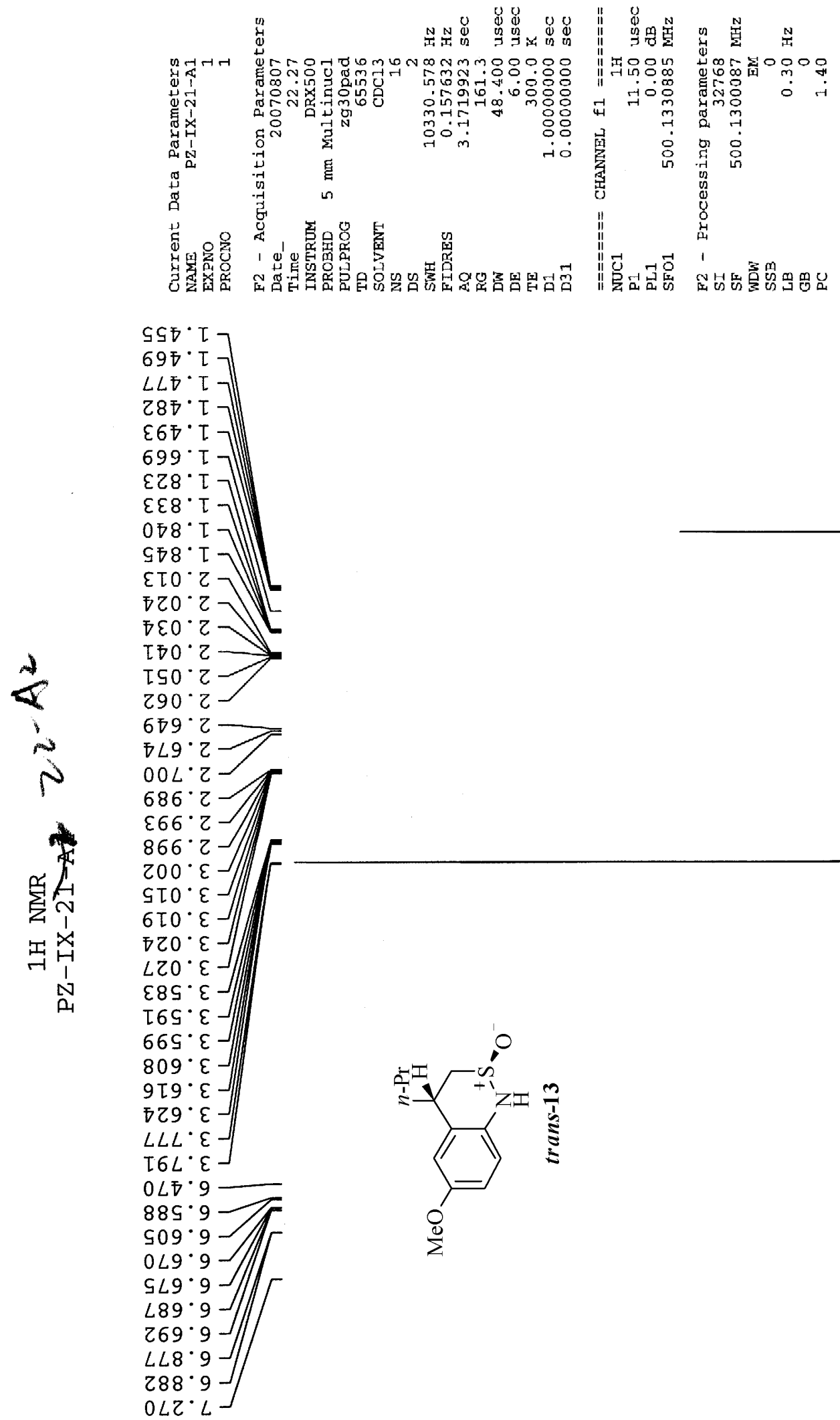

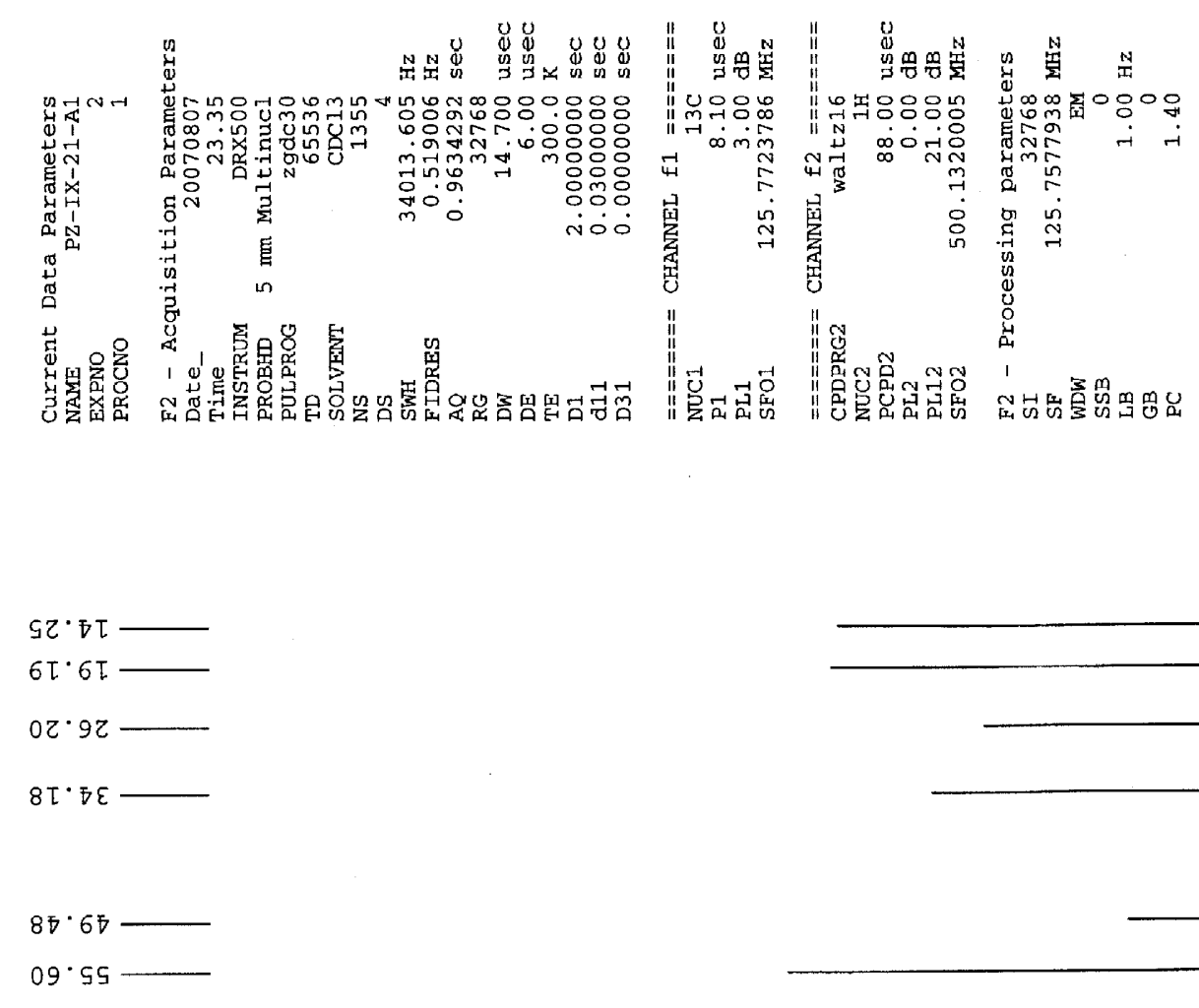

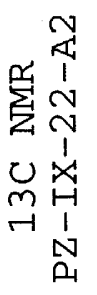
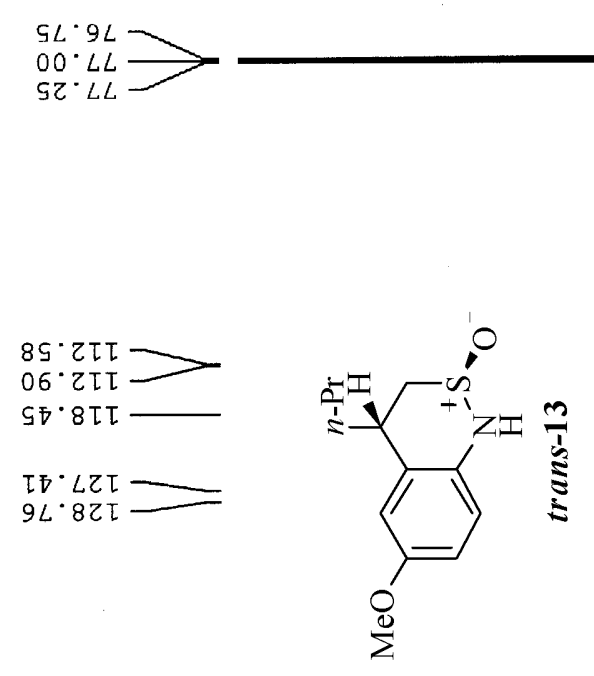

$L 6 \cdot \emptyset \subseteq \tau$ 


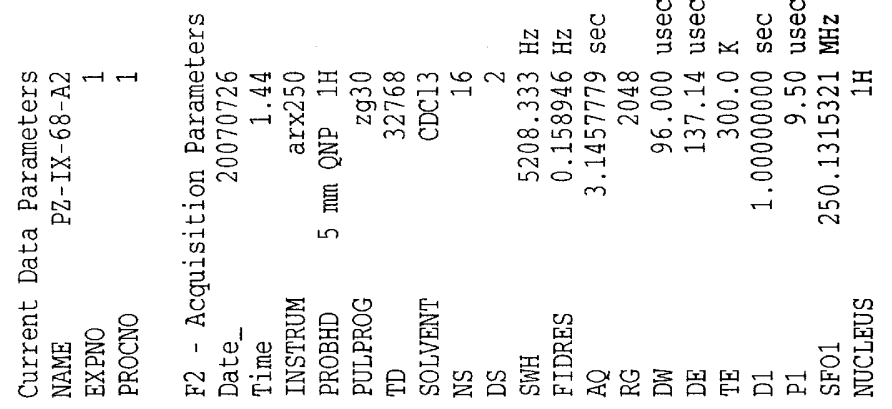

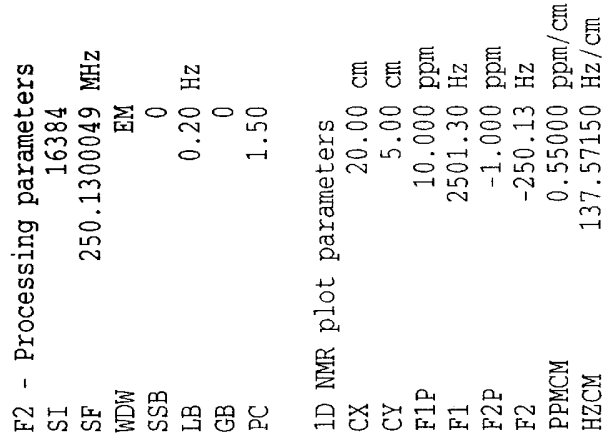

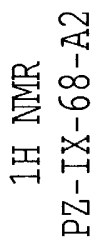
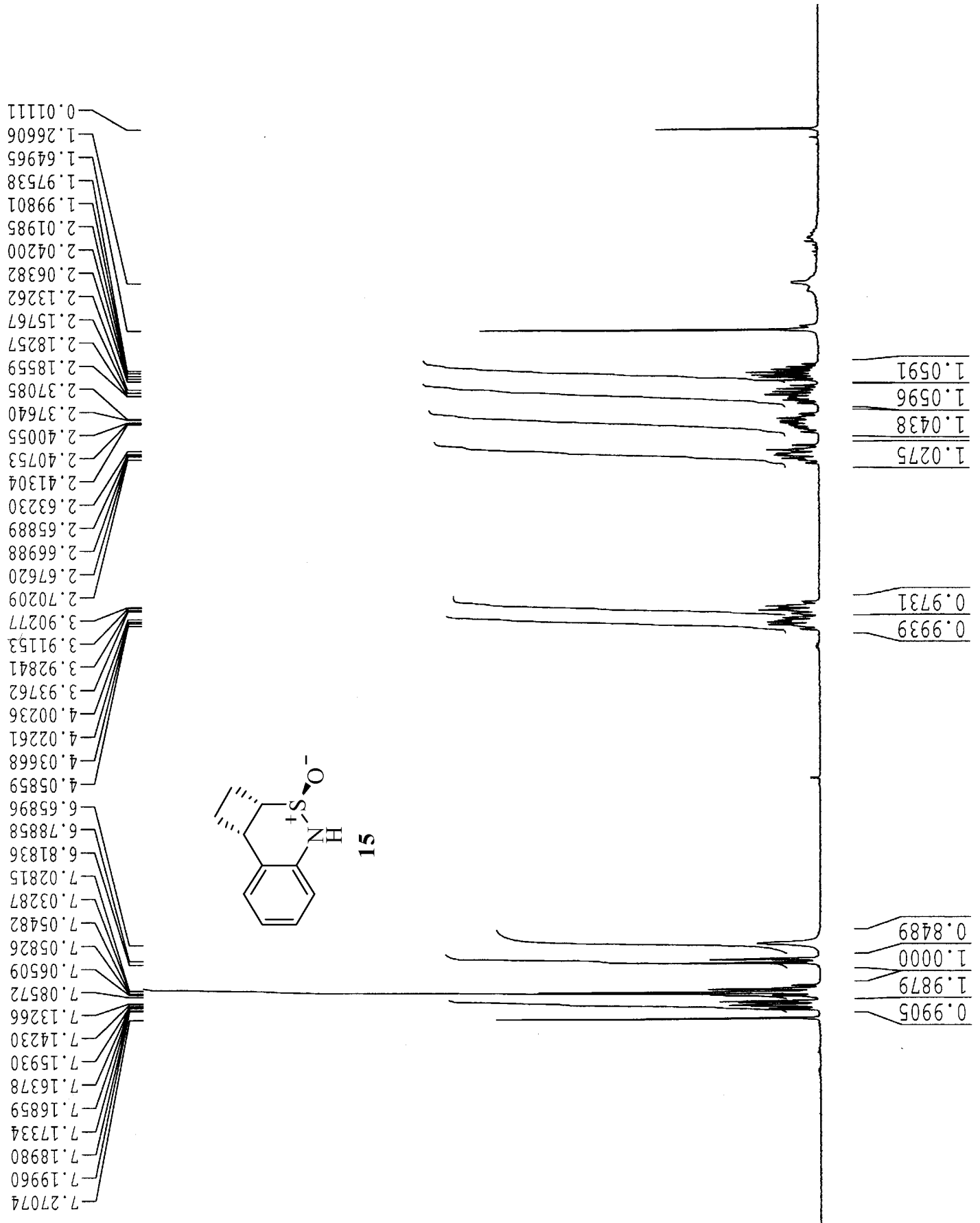

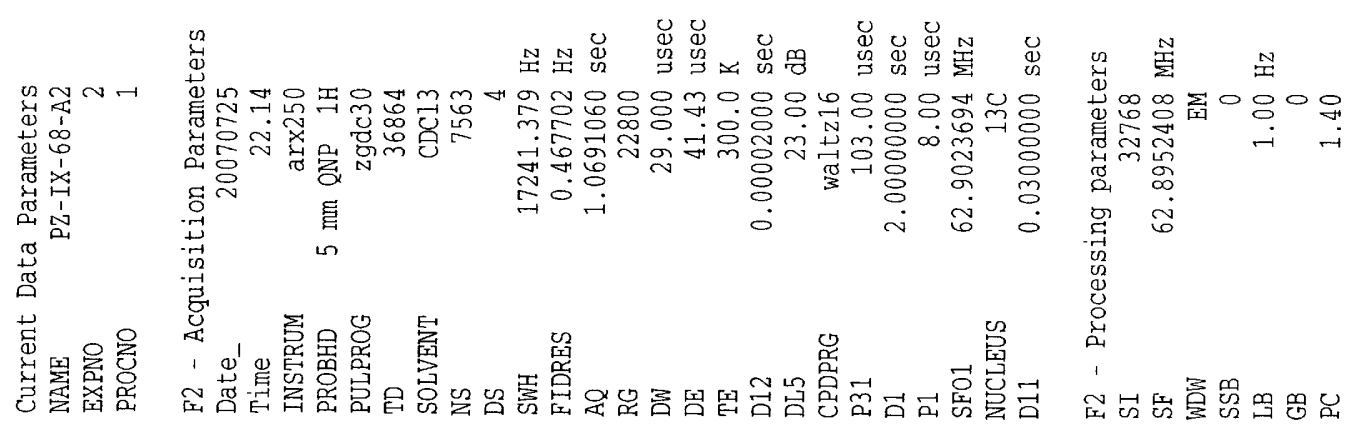

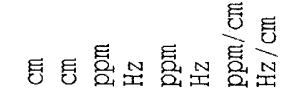

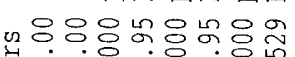

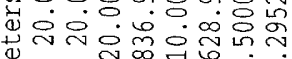
壳 苍

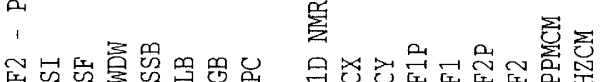

$802 \cdot 02$

$\varepsilon 98.82$

$982^{\circ}$ เE

LOL'LS

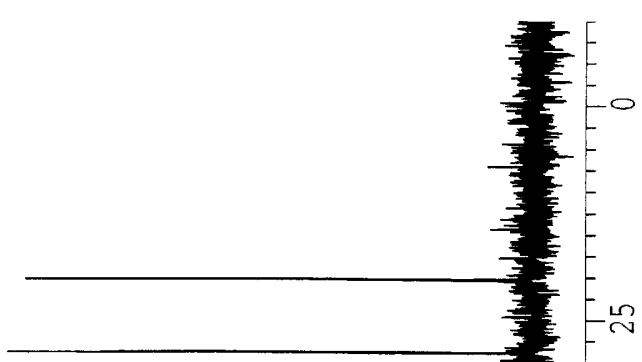

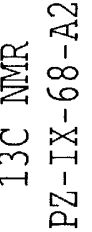

$560^{\circ} 9 \mathrm{~L}$

$200^{\circ} \mathrm{LL}$

IIS'LL
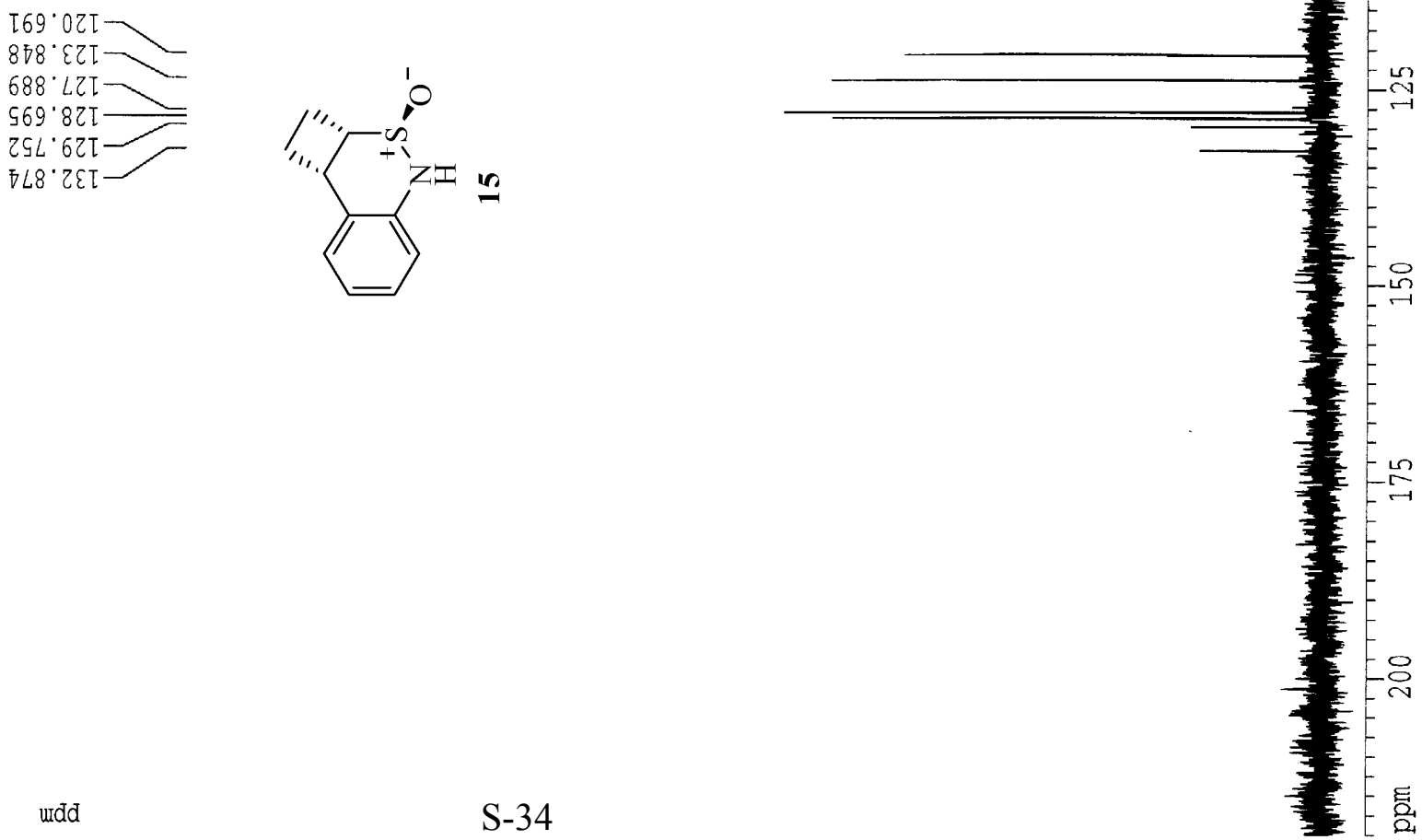


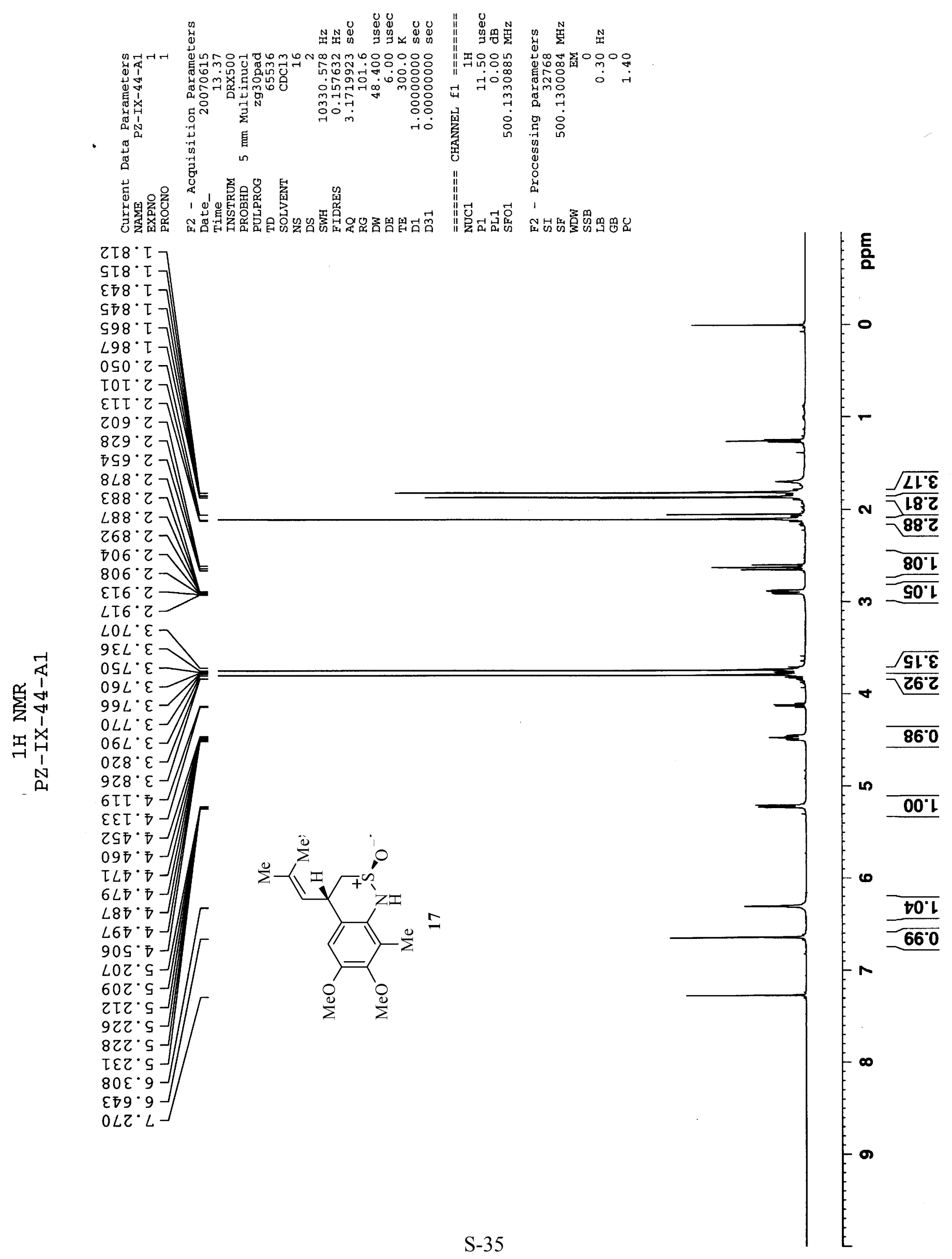



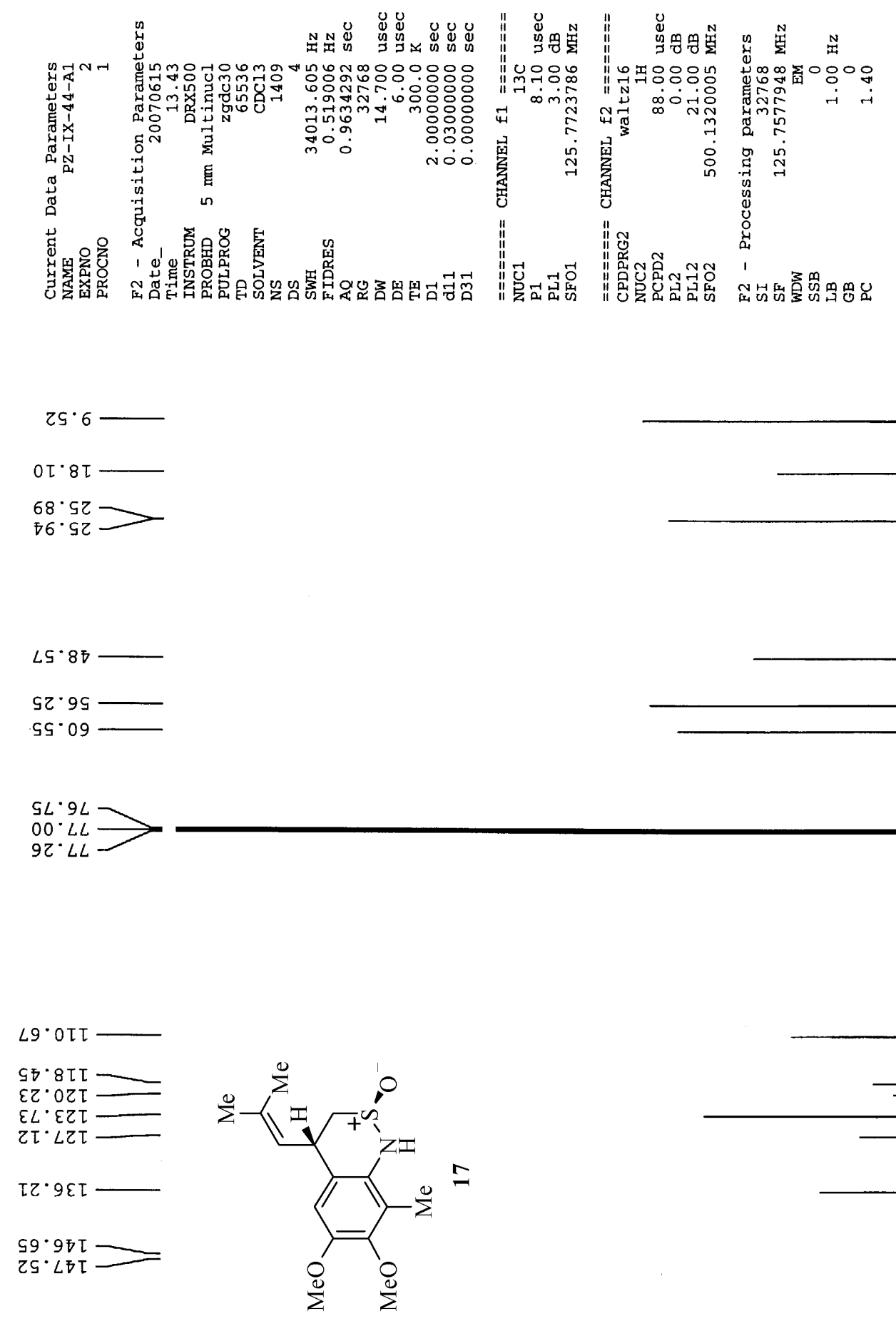

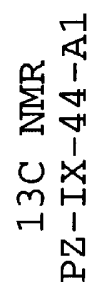




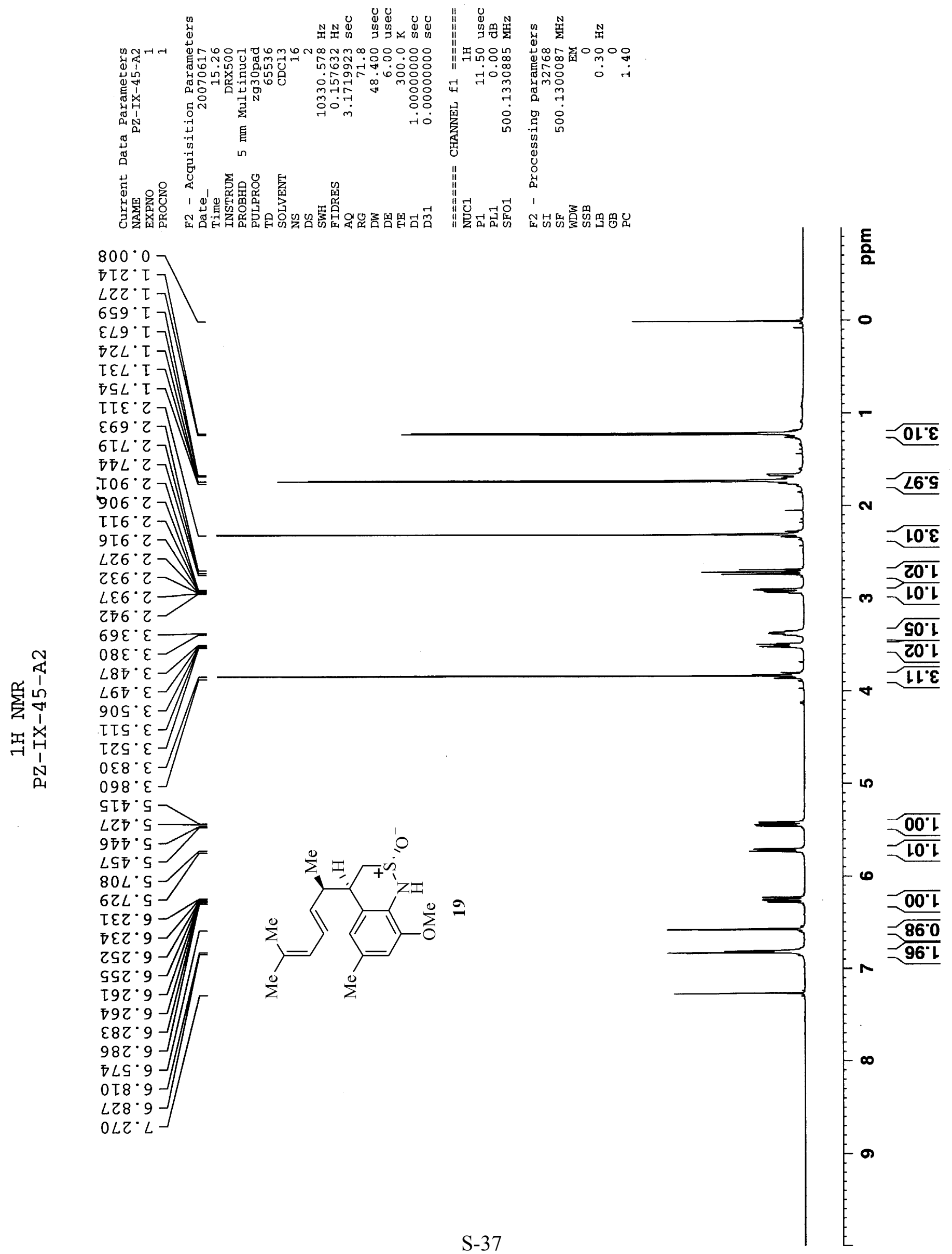




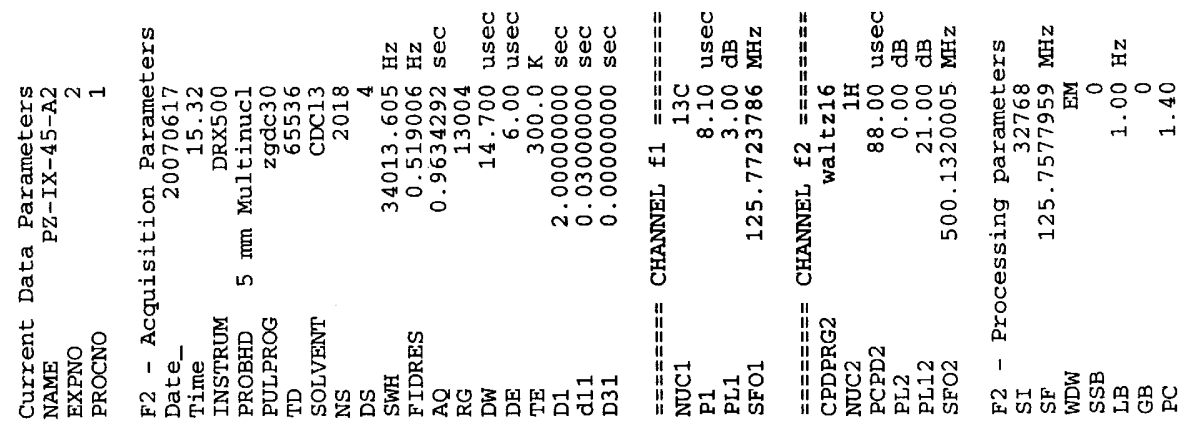

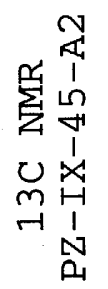
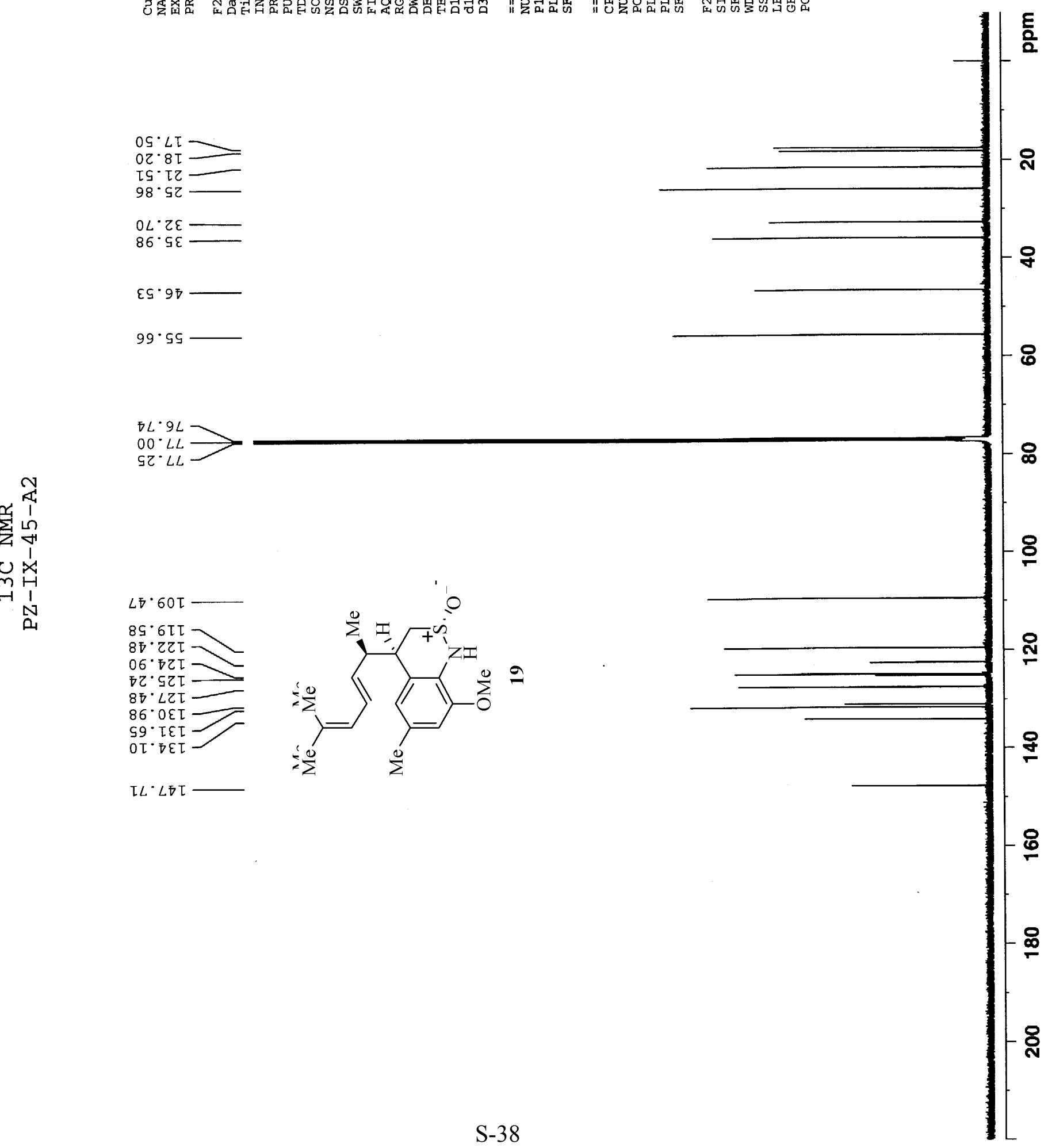


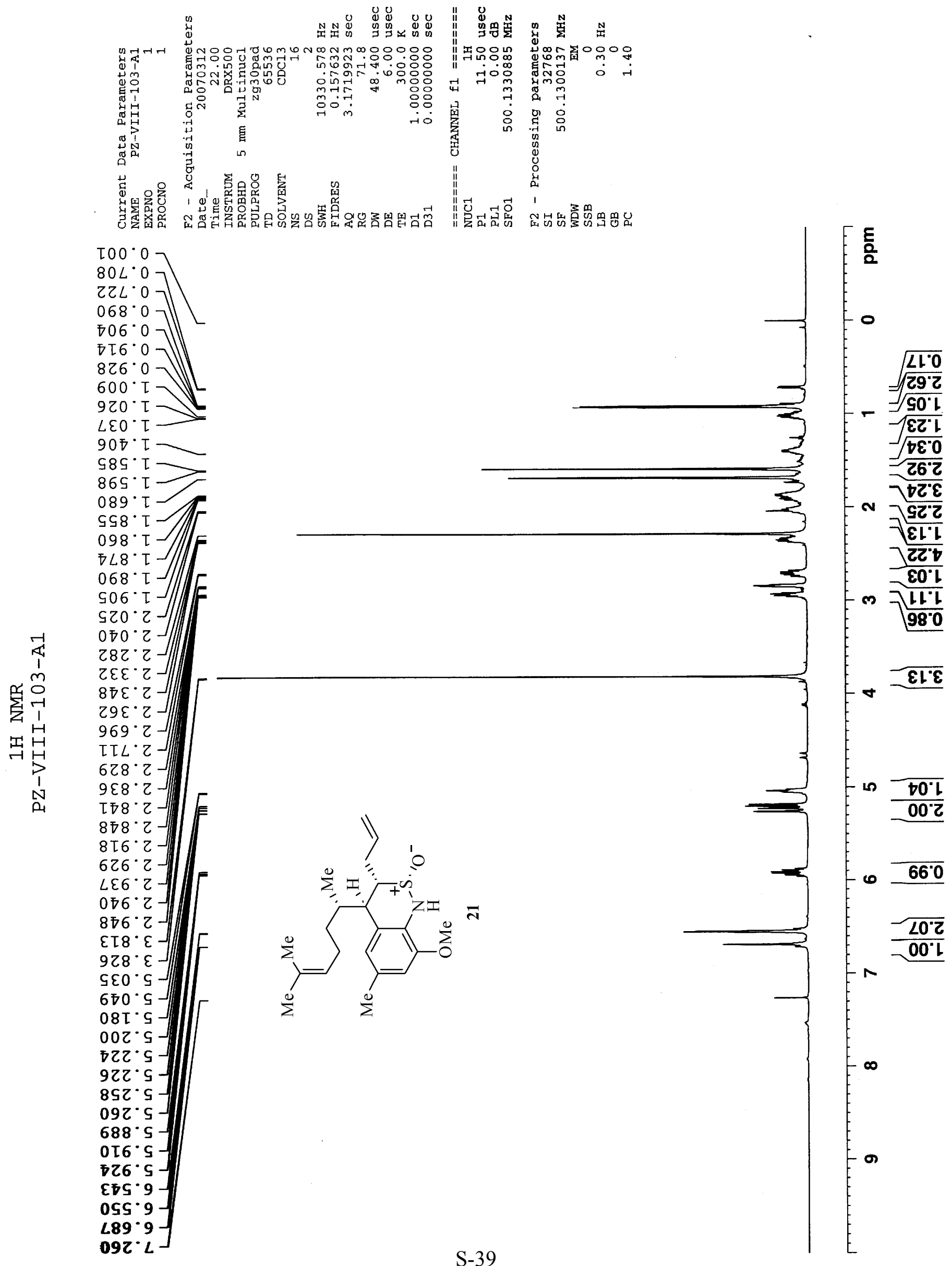




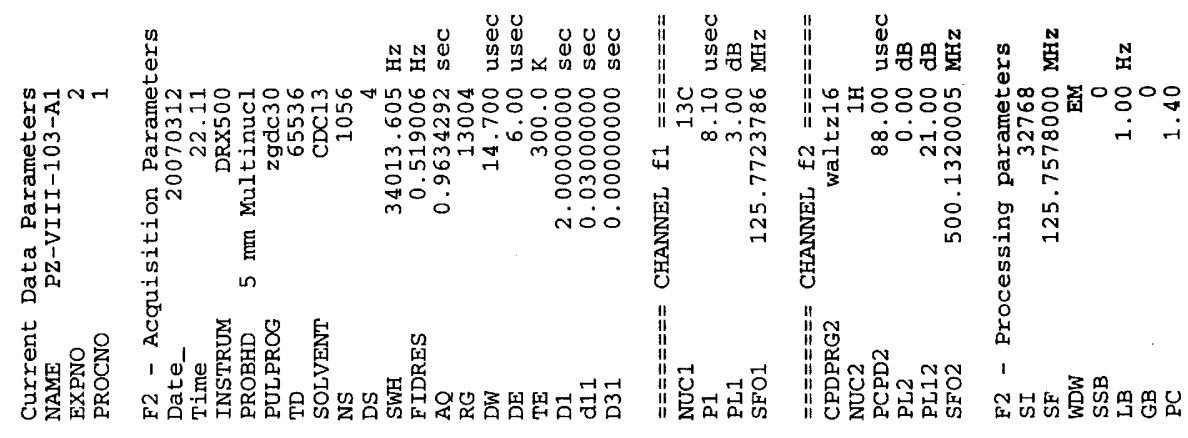

每

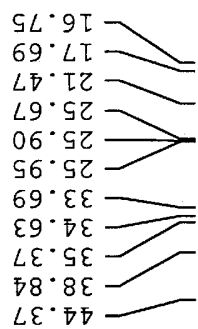

$\nabla \varsigma \cdot \varsigma 9$

$\varepsilon L \cdot 09$

'

1
1
1
1
1
1
1
1
1
1
1
1
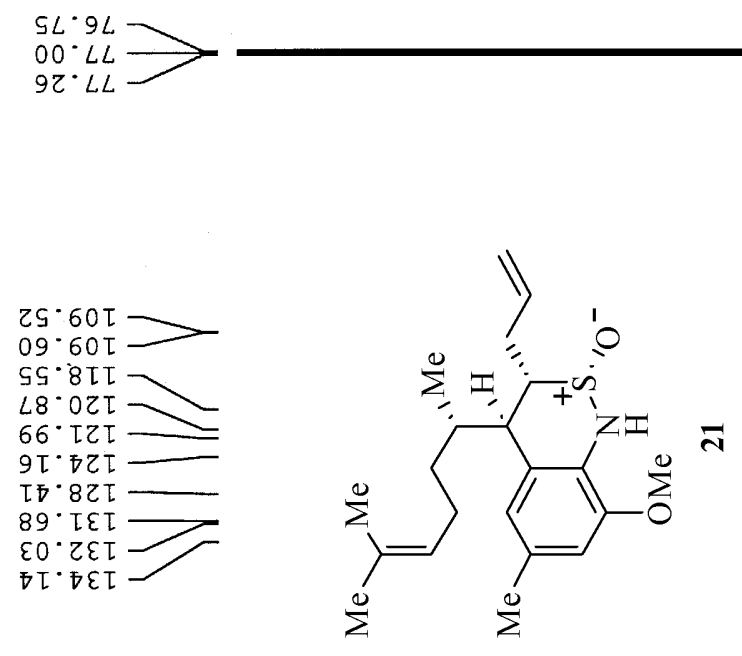

$\nabla \tau \cdot 6 \nabla \tau$

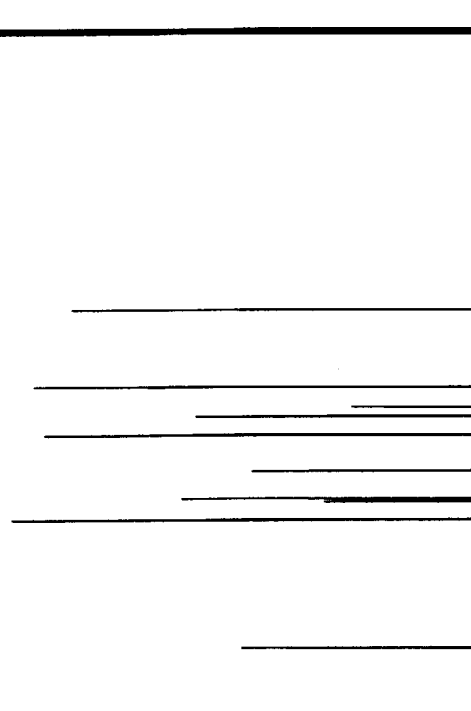




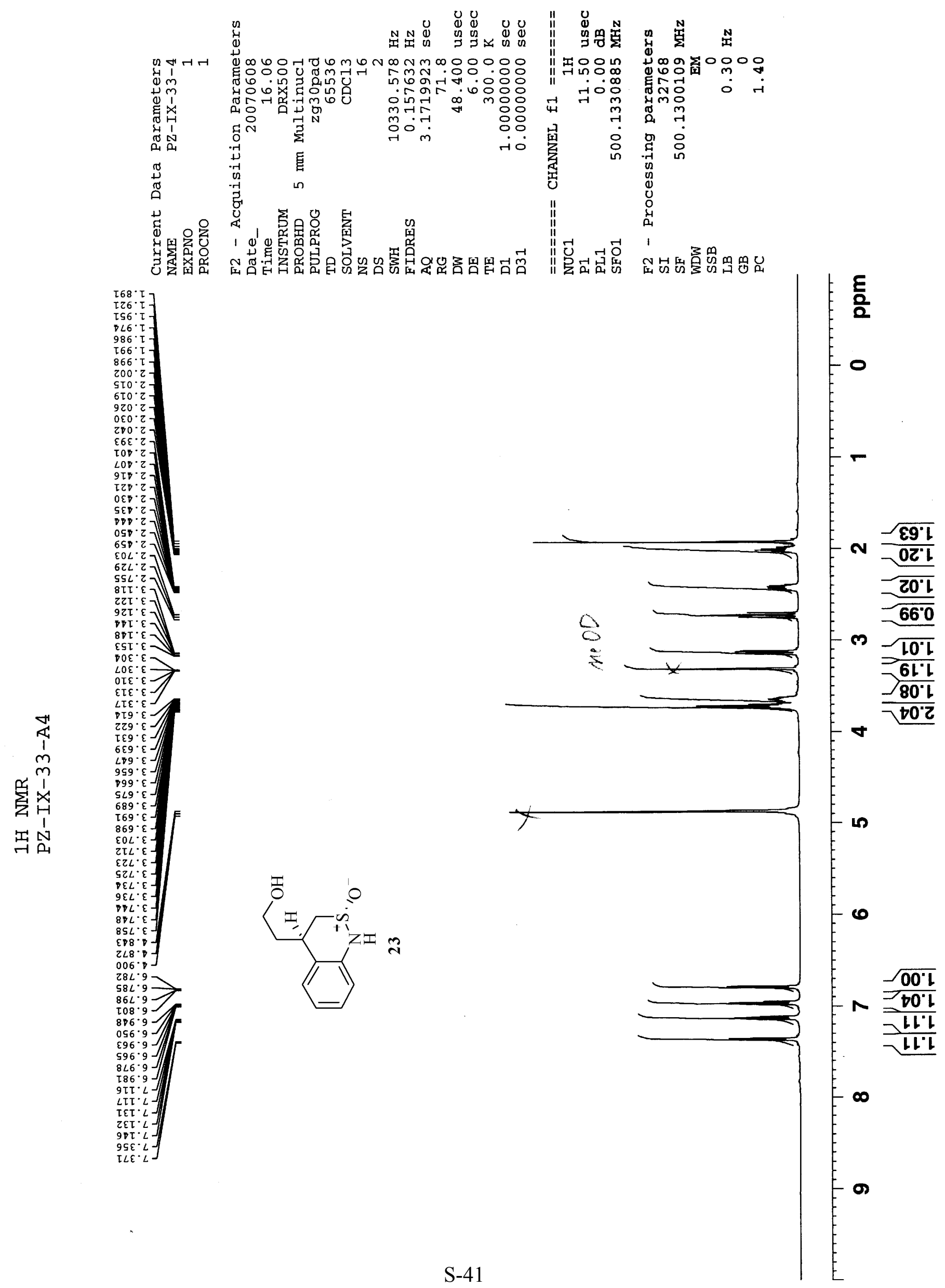



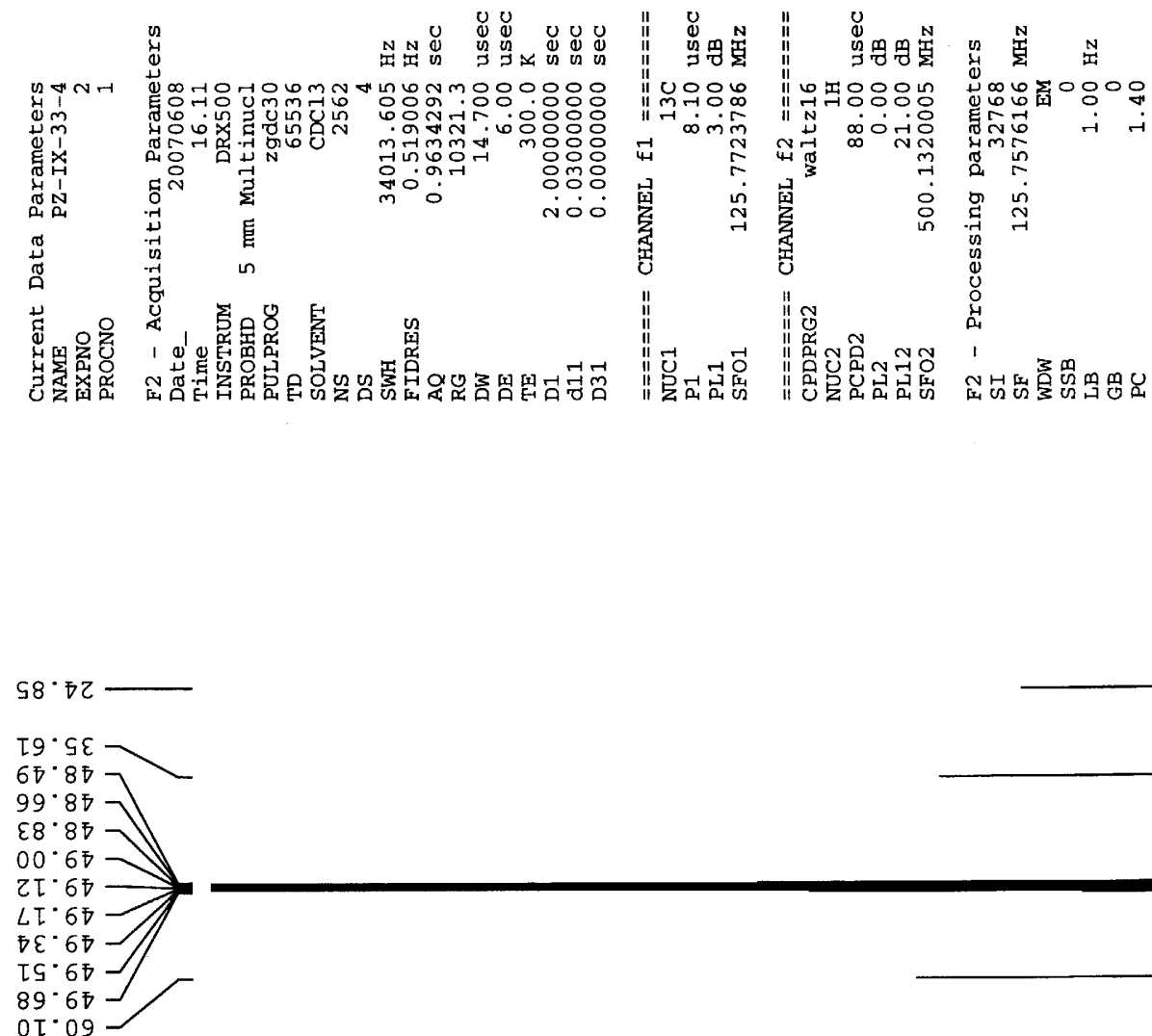

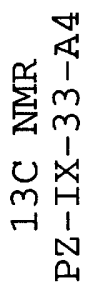

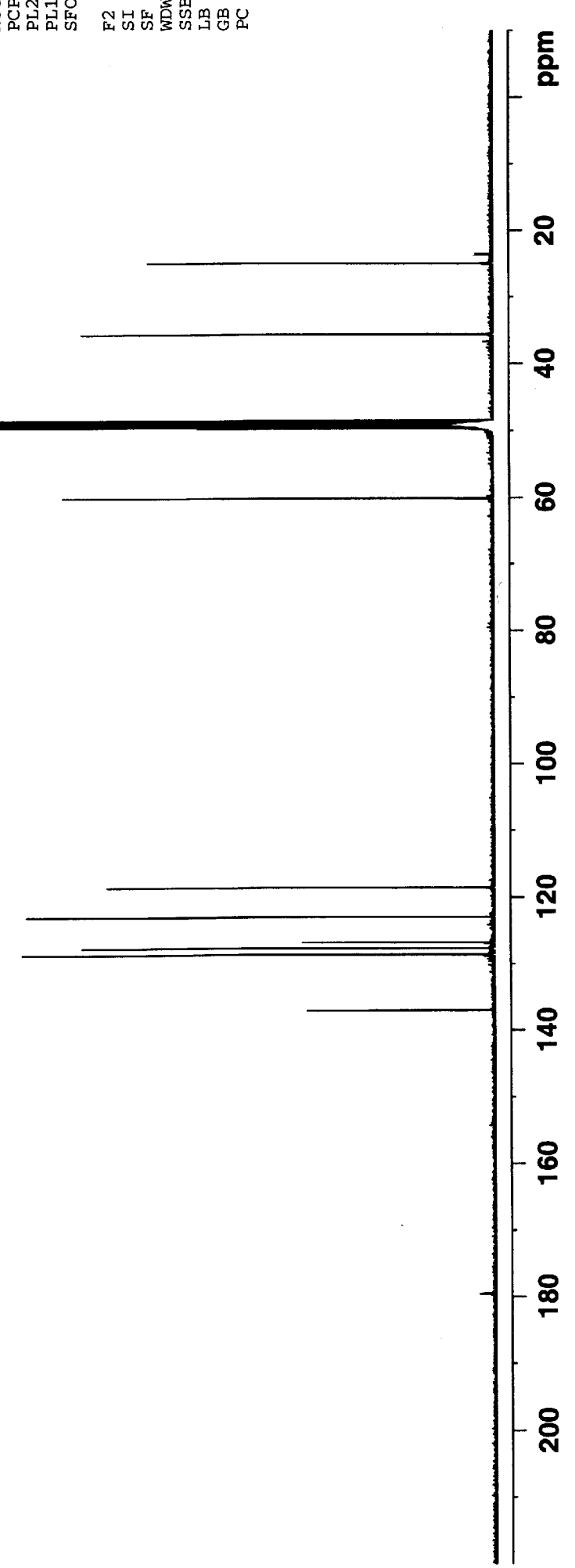

